\title{
Cocycles of $G$-Alexander biquandles and $G$-Alexander multiple conjugation biquandles
}

\author{
Atsushi Ishii, Masahide Iwakiri, Seiichi Kamada \\ Jieon Kim, Shosaku Matsuzaki, and Kanako Oshiro
}

February 27, 2020

\begin{abstract}
Biquandles and multiple conjugation biquandles are algebras which are related to links and handlebody-links in 3-space. Cocycles of them can be used to construct state-sum type invariants of links and handlebody-links. In this paper we discuss cocycles of a certain class of biquandles and multiple conjugation biquandles, which we call $G$-Alexander biquandles and $G$-Alexander multiple conjugation biquandles, with a relationship with group cocycles. We give a method to obtain a (biquandle or multiple conjugation biquandle) cocycle of them from a group cocycle.
\end{abstract}

\section{Introduction}

A quandle is an algebra related to knots and links (cf. 6, 16, 20]). Using a (quandle) 2-cocycle or 3-cocycle in the (co)homology theory of a quandle (cf. 3, 7, 8), we can construct an invariant of knots and links. A multiple conjugation quandle is introduced in [11. It is an algebra motivated by study of handlebody-links, which are isotopy classes of embedded handlebodies in 3space. Another notion called a qualgebra was introduced in [18, 19] which is related to trivalent graphs in 3 -space.

The notions of a quandle and a multiple conjugation quandle are generalized to biquandles (cf. 1, 4, 5, 17]) and multiple conjugation biquandles (cf. 13, 15), although the terminologies and definitions of such generalizations vary in the literature. Homology theory on biquandles is found in 1, 8 and homology theory on multiple conjugation biquandles is found in 14. In 14 we discussed the (co)homology theory of multiple conjugation biquandles and we provided a method of constructing an invariant of handlebody-links from a 2 or 3-cocycle. Thus, in order to construct such an invariant of links or handlebody-links, it is important to find a cocycle of a biquandle or a multiple conjugation biquandle. In this paper, we focus on a certain class of biquandles and multiple conjugation biquandles which we call $G$-Alexander biquandles and $G$-Alexander multiple conjugation biquandles.

The quandles associated to $G$-families of Alexander quandles 12 are an important class of quandles. In [21, Nosaka studied cocycles of a $G$-family $M$ of Alexander quandles, for a group $G$ and a $G$-module $M$, and gave a method of obtaining a cocycle of the associated quandle $M \times G$. For a given $G$-invariant group cocycle of the $G$-family $M$, we can construct a cocycle of the quandle 
$M \times G$ by using invariant theory. Our approach in this paper is an analogue of Nosaka's argument.

The paper is organized as follows. In Section 2, we review definitions related to the (co)homology of biquandles, multiple conjugation biquandles and groups. We also give the definition of $G$-Alexander biquandles and $G$-Alexander multiple conjugation biquandles. In Sections 3 and 4 we focus on $G$-Alexander biquandles $X=M \times G$ and cocycles of them. The case where the biquandle $X$ has the trivial $X$-set (or has $X$ itself as an $X$-set, resp.) is discussed in Section 3 (or 44). We show how to obtain a (birack) cocycle of the biquandle $X=M \times G$ from a group cocycle: Theorems 3.4 and 3.5 (Theorems 4.4 and 4.5 ). In Sections 5 and 6 we focus on $G$-Alexander multiple conjugation biquandles $X=\bigsqcup_{m \in M}(\{m\} \times G)=$ $M \times G$ and cocycles of them. The case where the biquandle $X$ has the trivial $X$ set (or has $X$ itself as an $X$-set, resp.) is discussed in Section 5 (or 6 ). We show how to obtain a (multiple conjugation biquandle) cocycle of the $G$-Alexander multiple conjugation biquandle from a group cocycle: Theorems 5.7 and 5.8 (Theorems 6.8 6.10 and 6.11). Since any $G$-invariant multilinear map provides a $G$-invariant group cocycle, we can obtain many cocycles.

This research is supported by JSPS KAKENHI Grant Numbers 16K17600, 18K03292 and 19H01788. It is also supported by Young Researchers Program through the National Research Foundation of Korea (NRF) funded by the Ministry of Education, Science and Technology (NRF-2018R1C1B6007021).

\section{Preliminaries}

In this section, we review definitions of biquandles and multiple conjugation biquandles, and the (co)homology of those and groups. In Subsection 2.2 we give the definition of $G$-Alexander biquandles and $G$-Alexander multiple conjugation biquandles.

\subsection{Biquandles and multiple conjugation biquandles (MCB)}

Definition 2.1 (17, 17)). A biquandle is a non-empty set $X$ with binary operations $\underline{*}, \bar{*}: X \times X \rightarrow X$ satisfying the following axioms.

(B1) For any $x \in X, x \underline{*} x=x \bar{*} x$.

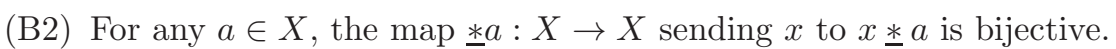

For any $a \in X$, the map $\bar{*} a: X \rightarrow X$ sending $x$ to $x \mp a$ is bijective.

The map $S: X \times X \rightarrow X \times X$ defined by $S(x, y)=(y \bar{\star} x, x$ ㄴ $y)$ is bijective.

(B3) For any $x, y, z \in X$,

$$
\begin{aligned}
& (x \text { ㄸy } y) \text { }(z \text { } y)=(x \text { 느 } z) \text { }(y \bar{*} z), \\
& (x \underline{*} y) \bar{F}(z \text { } y)=(x \bar{*} z) \text { * }(y \bar{*} z) \text {, } \\
& (x \bar{*} y) \bar{*}(z \bar{*} y)=(x \bar{*} z) \bar{*}(y \underline{*} z) .
\end{aligned}
$$

A birack is a non-empty set $X$ with binary operations $\underline{*}, \bar{*}: X \times X \rightarrow X$ satisfying (B2) and (B3). 
Definition 2.2. For a biquandle (or a birack) $X$, an $X$-set is a non-empty set $Y$ with a map $*: Y \times X \rightarrow Y$ satisfying the following axioms.

- For any $y \in Y$ and $a, b \in X,(y * a) *(b * a)=(y * b) *(a * b)$.

- For any $x \in X$, the map $* x: Y \rightarrow Y ; y \mapsto y * x$ is bijective.

Let $X$ be the disjoint union of groups $G_{\lambda}(\lambda \in \Lambda)$. We denote by $G_{a}$ the group $G_{\lambda}$ to which $a \in X$ belongs. We denote by $e_{\lambda}$ the identity of $G_{\lambda}$.

Definition 2.3 (13). A multiple conjugation biquandle is a non-empty set $X$ which is the disjoint union of groups $G_{\lambda}(\lambda \in \Lambda)$ with binary operations *, $\bar{*}: X \times X \rightarrow X$ satisfying the following axioms.

- For any $x, y, z \in X$,

$$
\begin{aligned}
& (x \underline{*} y) \text { }(z \underline{*} y)=(x \text { 느 } z) \text { }(y \bar{*} z), \\
& (x \text { } y) \text { * }(z \text { } y)=(x \bar{*} z) \text { }(y \text { * } z), \\
& (x \bar{*} y) \mp(z \bar{*} y)=(x \bar{*} z) \text { ₹( }(y \underline{*} z) \text {. }
\end{aligned}
$$

- For any $a, x \in X$, the maps $\underline{* a}: G_{x} \rightarrow G_{x \underline{a}}$ and $\bar{*} a: G_{x} \rightarrow G_{x \bar{\star} a}$ are group homomorphisms.

- For any $a, b \in G_{\lambda}$ and $x \in X$,

$$
\begin{aligned}
& x \underline{*} a b=(x \underline{*} a) \text { * }(b \bar{*} a), \quad x \text { 늣 } e_{\lambda}=x, \\
& x \bar{*} a b=(x \bar{*} a) \bar{*}(b \bar{*} a), \quad x \bar{*} e_{\lambda}=x, \\
& a^{-1} b \bar{*} a=b a^{-1} \underline{*} a .
\end{aligned}
$$

A multiple conjugation biquandle $\left(X=\bigsqcup_{\lambda \in \Lambda} G_{\lambda}, \underline{*}, \bar{*}\right)$ is regarded as a biquandle $(X, \underline{*}, \bar{*})$ by forgetting the decomposition $X=\bigsqcup_{\lambda \in \Lambda} G_{\lambda}$ and the group structure of each $G_{\lambda}$.

Definition 2.4. For a multiple conjugation biquandle $X=\bigsqcup_{\lambda \in \Lambda} G_{\lambda}$, an $X$-set is a non-empty set $Y$ with a map $*: Y \times X \rightarrow Y$ satisfying the following axioms.

- For any $y \in Y$ and $a, b \in G_{\lambda}, y * e_{\lambda}=y, y *(a b)=(y * a) *(b * a)$, where $e_{\lambda}$ is the identity of $G_{\lambda}$.

- For any $y \in Y$ and $a, b \in X,(y * a) *(b \mp a)=(y * b) *(a$ $b)$.

Any singleton set $\left\{y_{0}\right\}$ is also an $X$-set with the map $*$ defined by $y_{0} * x=y_{0}$ for $x \in X$, which is called a trivial $X$-set. Any multiple conjugation biquandle $X$ itself is an $X$-set with the map $*$ or $*$.

\section{$2.2 \quad G$-Alexander biquandles and $G$-Alexander multiple con- jugation biquandles}

Definition 2.5. Let $R$ be a ring, $G$ a group and $M$ a right $R[G]$-module, where $R[G]$ is the group ring of $G$ over $R$. Let $\varphi: G \rightarrow Z(G)$ be a group homomorphism, where $Z(G)$ denotes the center of $G$. We define binary operations $\underline{*}$ and $\bar{*}:(M \times G)^{2} \rightarrow M \times G$ by

$$
\begin{aligned}
& (m, g) \underline{*}(n, h):=\left(m h+n(\varphi(h)-h), h^{-1} g h\right), \\
& (m, g) \Psi(n, h):=(m \varphi(h), g) .
\end{aligned}
$$


Then, $(M \times G, \underline{*}, \bar{*})$ is a biquandle (cf.[15]), which we call the $G$-Alexander biquandle of $(M, \varphi)$, and $\left(M \times G=\bigsqcup_{m \in M}(\{m\} \times G), *, *\right)$ is a multiple conjugation biquandle by

$$
(m, g)(m, h):=(m, g h)
$$

for any $m \in M$ and any $g, h \in G$ (cf.[15]), which we call the $G$-Alexander multiple conjugation biquandle of $(M, \varphi)$.

Unless otherwise stated, we suppose that the $\operatorname{ring} R$ is $\mathbb{Z}$.

Remark 2.6. In general, for a given $G$-family of biquandles, one can associate a biquandle called the associated biquandle of the G-family of biquandles (15), and a multiple conjugation biquandle called the associated multiple conjugation biquandle of the G-family of biquandles or the partially multiplicative biquandle associated to the G-family of biquandles ([15]). The $G$-Alexander biquandle (or the $G$-Alexander multiple conjugation biquandle) in the definition above is the associated biquandle (or the associated multiple conjugation biquandle) of a "G-family of Alexander biquandle" in the sense of [15].

\subsection{The (co)homology of biquandles}

In this subsection, we review the birack chain complex for a biquandle.

Let $X$ be a biquandle (or more generally a birack) and $Y$ an $X$-set. Let $C_{n}^{\mathrm{BR}}(X ; \mathbb{Z})_{Y}$ denote the free abelian group generated by the elements $\boldsymbol{x}=$ $\left(y, x_{1}, \ldots, x_{n}\right) \in Y \times X^{n}$ if $n \geq 1$, and $C_{n}^{\mathrm{BR}}(X ; \mathbb{Z})_{Y}=0$ otherwise. (The superscripts BR means birack.)

For integers $n$ and $i$ such that $n \geq 2$ and $1 \leq i \leq n$ and for any element $\boldsymbol{x}=\left(y, x_{1}, \ldots, x_{i}, \ldots, x_{n}\right) \in Y \times X^{n}$, we write

$$
\begin{aligned}
\left(\boldsymbol{x}^{i-1}, \boldsymbol{x}_{i+1}\right) & :=\left(y, x_{1}, \ldots, x_{i-1}, x_{i+1}, \ldots, x_{n}\right) \text { and } \\
\left(\boldsymbol{x}^{i-1} \underline{*} x_{i}, \boldsymbol{x}_{i+1} \bar{*} x_{i}\right) & :=\left(y * x, x_{1} \underline{*} x_{i}, \ldots, x_{i-1} \underline{*} x_{i}, x_{i+1} \bar{*} x_{i}, \ldots, x_{n} x_{i}\right),
\end{aligned}
$$

where these elements belong to $Y \times X^{n-1}$.

Define a boundary map $\partial_{n}^{\mathrm{BR}}: C_{n}^{\mathrm{BR}}(X ; \mathbb{Z})_{Y} \rightarrow C_{n-1}^{\mathrm{BR}}(X ; \mathbb{Z})_{Y}$ by

$$
\partial_{n}^{\mathrm{BR}}(\boldsymbol{x}):=\sum_{i=1}^{n}(-1)^{i-1}\left\{\left(\boldsymbol{x}^{i-1}, \boldsymbol{x}_{i+1}\right)-\left(\boldsymbol{x}^{i-1} \underline{*} x_{i}, \boldsymbol{x}_{i+1} \bar{*} x_{i}\right)\right\}
$$

for $n \geq 2$, and define $\partial_{n}^{\mathrm{BR}}=0$ for $n \leq 1$.

For example, we have

$$
\begin{aligned}
& \partial_{4}^{\mathrm{BR}}\left(y, x_{1}, x_{2}, x_{3}, x_{4}\right)=+\left\{\left(y, x_{2}, x_{3}, x_{4}\right)-\left(y * x_{1}, x_{2} \mp x_{1}, x_{3} \mp x_{1}, x_{4} \mp x_{1}\right)\right\} \\
& -\left\{\left(y, x_{1}, x_{3}, x_{4}\right)-\left(y * x_{2}, x_{1} \underline{*} x_{2}, x_{3} \mp x_{2}, x_{4} \mp x_{2}\right)\right\} \\
& +\left\{\left(y, x_{1}, x_{2}, x_{4}\right)-\left(y * x_{3}, x_{1} \underset{*}{*} x_{3}, x_{2} * x_{3}, x_{4} \bar{*} x_{3}\right)\right\} \\
& -\left\{\left(y, x_{1}, x_{2}, x_{3}\right)-\left(y * x_{4}, x_{1} \underline{*} x_{4}, x_{2} \underline{*} x_{4}, x_{3} \underline{*} x_{4}\right)\right\} .
\end{aligned}
$$

Proposition 2.7 (cf. [1]). $C_{*}^{\mathrm{BR}}(X ; \mathbb{Z})_{Y}:=\left(C_{n}^{\mathrm{BR}}(X ; \mathbb{Z})_{Y}, \partial_{n}^{\mathrm{BR}}\right)_{n \in \mathbb{Z}}$ is a chain complex.

The birack chain complex of $X$ with an $X$-set $Y$ is the chain complex $C_{*}^{\mathrm{BR}}(X ; \mathbb{Z})_{Y}$. It determines the birack homology group $H_{n}^{\mathrm{BR}}(X ; \mathbb{Z})_{Y}$ and the 
cohomology group $H_{\mathrm{BR}}^{n}(X ; \mathbb{Z})_{Y}$. For an abelian group $A$, the chain and cochain complexes $C_{*}^{\mathrm{BR}}(X ; A)_{Y}$ and $C_{\mathrm{BR}}^{*}(X ; A)_{Y}$ are also defined in the ordinary way. We denote by $H_{n}^{\mathrm{BR}}(M ; A)_{Y}$ and $H_{\mathrm{BR}}^{n}(X ; A)_{Y}$ its homology and cohomology groups. A cocycle of $C_{\mathrm{BR}}^{*}(X ; A)_{Y}$ is called a birack cocycle with $Y$ of the biquandle $X$ with $A$. We omit $Y$, that is, we write $C_{*}^{\mathrm{BR}}(X ; \mathbb{Z}):=C_{*}^{\mathrm{BR}}(X ; \mathbb{Z})_{Y}$ if $Y$ is the trivial $X$-set.

\subsection{The (co)homology of multiple conjugation biquandles}

We review chain complexes $P_{*}(X ; \mathbb{Z})_{Y}, D_{*}(X ; \mathbb{Z})_{Y}$ and $C_{*}(X ; \mathbb{Z})_{Y}$ defined in 14 for multiple conjugation biquandles $X$ with $X$-set $Y$. Refer to 14 for a geometric interpretation of the chain complexes and an application in knot theory. These chain complexes are an analogy of chain complexes defined in [2] for multiple conjugation quandles.

Let $X=\bigsqcup_{\lambda \in \Lambda} G_{\lambda}$ be a multiple conjugation biquandle and $Y$ an $X$-set of the MCB $X$. For $n \geq 0$, let $P_{n}(X ; \mathbb{Z})_{Y}$ denote the free abelian group generated by the elements

$$
\langle y\rangle\left\langle\boldsymbol{x}_{1}\right\rangle \cdots\left\langle\boldsymbol{x}_{k}\right\rangle \in \bigcup_{n_{1}+\cdots+n_{k}=n} Y \times \prod_{i=1}^{k} \bigcup_{\lambda \in \Lambda} G_{\lambda}^{n_{i}},
$$

where $\left\langle\boldsymbol{x}_{i}\right\rangle$ stands for an element $\left\langle x_{i 1}, \ldots, x_{i n_{i}}\right\rangle \in \bigcup_{\lambda \in \Lambda} G_{\lambda}^{n_{i}}$. For $n<0$, let $P_{n}(X ; \mathbb{Z})_{Y}=0$. We write

$$
\begin{aligned}
& \left\langle\boldsymbol{x}_{i} \text { * } x\right\rangle:=\left\langle x_{i 1} \mp x, x_{i 2}{ }^{*} x, \ldots, x_{i n_{i}} \bar{*} x\right\rangle \text { and } \\
& \left\langle\boldsymbol{x}_{i} \underline{*} x\right\rangle:=\left\langle x_{i 1} \underline{*} x, x_{i 2} \underline{*} x, \ldots, x_{i n_{i}} \underline{*} x\right\rangle
\end{aligned}
$$

for an element $x \in X$ and an element $\left\langle\boldsymbol{x}_{i}\right\rangle=\left\langle x_{i 1}, \ldots, x_{i n_{i}}\right\rangle \in \bigcup_{\lambda \in \Lambda} G_{\lambda}^{n_{i}}$.

Define a boundary map $\partial_{n}: P_{n}(X ; \mathbb{Z})_{Y} \rightarrow P_{n-1}(X ; \mathbb{Z})_{Y}$ by

$$
\begin{aligned}
\partial_{n}\left(\langle y\rangle\left\langle\boldsymbol{x}_{1}\right\rangle \cdots\left\langle\boldsymbol{x}_{k}\right\rangle\right):=\sum_{i=1}^{k}(-1)^{n_{1}+\cdots+n_{i-1}}\{ \\
\quad\left\langle y * x_{i 1}\right\rangle\left\langle\boldsymbol{x}_{1} \text { } x_{i 1}\right\rangle \cdots\left\langle\boldsymbol{x}_{i-1} * x_{i 1}\right\rangle\left\langle\widetilde{\boldsymbol{x}}_{i} \bar{*} x_{i 1}\right\rangle\left\langle\boldsymbol{x}_{i+1} \bar{*} x_{i 1}\right\rangle \cdots\left\langle\boldsymbol{x}_{k} \bar{*} x_{i 1}\right\rangle \\
\left.\quad+\sum_{j=1}^{n_{i}}(-1)^{j}\langle y\rangle\left\langle\boldsymbol{x}_{1}\right\rangle \cdots\left\langle\boldsymbol{x}_{i-1}\right\rangle\left\langle x_{i 1}, \ldots, x_{i(j-1)}, x_{i(j+1)}, \ldots, x_{i n_{i}}\right\rangle\left\langle\boldsymbol{x}_{i+1}\right\rangle \cdots\left\langle\boldsymbol{x}_{k}\right\rangle\right\}
\end{aligned}
$$

when $n=n_{1}+\cdots+n_{k} \geq 1$, and define $\partial_{n}=0$ if $n<0$, where

$$
\left\langle\widetilde{\boldsymbol{x}}_{i} \bar{*} x_{i 1}\right\rangle:=\left\langle x_{i 1}^{-1} x_{i 2} \bar{*} x_{i 1}, x_{i 1}^{-1} x_{i 3} \bar{*} x_{i 1}, \ldots, x_{i 1}^{-1} x_{i\left(n_{i}-1\right)} \bar{*} x_{i 1}, x_{i 1}^{-1} x_{i n_{i}} \bar{*} x_{i 1}\right\rangle .
$$


For example, the boundary maps are computed as follows.

$$
\begin{aligned}
& \partial_{3}\left(\langle y\rangle\left\langle x_{1}\right\rangle\left\langle x_{2}\right\rangle\left\langle x_{3}\right\rangle\right) \\
& =\left\langle y * x_{1}\right\rangle\left\langle x_{2} \approx x_{1}\right\rangle\left\langle x_{3} \mp x_{1}\right\rangle-\langle y\rangle\left\langle x_{2}\right\rangle\left\langle x_{3}\right\rangle \\
& -\left\langle y * x_{2}\right\rangle\left\langle x_{1} \underline{*} x_{2}\right\rangle\left\langle x_{3} \mp x_{2}\right\rangle+\langle y\rangle\left\langle x_{1}\right\rangle\left\langle x_{3}\right\rangle \\
& +\left\langle y * x_{3}\right\rangle\left\langle x_{1} \text { * } x_{3}\right\rangle\left\langle x_{2} \text { * } x_{3}\right\rangle-\langle y\rangle\left\langle x_{1}\right\rangle\left\langle x_{2}\right\rangle, \\
& \partial_{3}\left(\langle y\rangle\left\langle x_{1}\right\rangle\left\langle x_{2}, x_{3}\right\rangle\right) \\
& =\left\langle y * x_{1}\right\rangle\left\langle x_{2} \mp x_{1}, x_{3} \mp x_{1}\right\rangle-\langle y\rangle\left\langle x_{2}, x_{3}\right\rangle-\left\langle y * x_{2}\right\rangle\left\langle x_{1} * x_{2}\right\rangle\left\langle x_{2}^{-1} x_{3} \mp x_{2}\right\rangle \\
& +\langle y\rangle\left\langle x_{1}\right\rangle\left\langle x_{3}\right\rangle-\langle y\rangle\left\langle x_{1}\right\rangle\left\langle x_{2}\right\rangle \\
& \partial_{3}\left(\langle y\rangle\left\langle x_{1}, x_{2}\right\rangle\left\langle x_{3}\right\rangle\right) \\
& =\left\langle y * x_{1}\right\rangle\left\langle x_{1}^{-1} x_{2} * x_{1}\right\rangle\left\langle x_{3} x^{*} x_{1}\right\rangle-\langle y\rangle\left\langle x_{2}\right\rangle\left\langle x_{3}\right\rangle \\
& +\langle y\rangle\left\langle x_{1}\right\rangle\left\langle x_{3}\right\rangle+\left\langle y * x_{3}\right\rangle\left\langle x_{1} \underline{*} x_{3}, x_{2} \underline{*} x_{3}\right\rangle-\langle y\rangle\left\langle x_{1}, x_{2}\right\rangle, \\
& \partial_{3}\left(\langle y\rangle\left\langle x_{1}, x_{2}, x_{3}\right\rangle\right) \\
& =\left\langle y * x_{1}\right\rangle\left\langle x_{1}^{-1} x_{2} x_{1}, x_{1}^{-1} x_{3} \mp x_{1}\right\rangle-\langle y\rangle\left\langle x_{2}, x_{3}\right\rangle+\langle y\rangle\left\langle x_{1}, x_{3}\right\rangle-\langle y\rangle\left\langle x_{1}, x_{2}\right\rangle .
\end{aligned}
$$

Remark 2.8. The notations of this paper are different from that of the paper [14, where we used more notations in order to prove propositions clearly.

Proposition $2.9\left([14) . P_{*}(X ; \mathbb{Z})_{Y}:=\left(P_{n}(X ; \mathbb{Z})_{Y}, \partial_{n}\right)_{n \in \mathbb{Z}}\right.$ is a chain complex.

For positive integers $s$ and $t$, we put

$$
M(s, t):=\{\mu:\{1, \ldots, s\} \rightarrow\{1, \ldots, t\} \mid i<j \Rightarrow \mu(i)<\mu(j)\} .
$$

For any map $\mu \in M(s, t)$ and any integer $j \in \mathbb{Z}$, we define $\lfloor j ; \mu\rfloor \in\{0,1, \ldots, s\}$ as $\lfloor j ; \mu\rfloor:=\max \{\ell \mid \mu(\ell) \leq j\}$.

For any $\lambda \in \Lambda$ and for elements $a_{1}, \ldots, a_{s}, b_{1}, \ldots, b_{t} \in G_{\lambda}$, put $\langle\boldsymbol{a}\rangle:=$ $\left\langle a_{1}, \ldots, a_{s}\right\rangle$ and $\langle\boldsymbol{b}\rangle:=\left\langle b_{1}, \ldots, b_{t}\right\rangle$. We set

$$
\begin{aligned}
\langle\langle\boldsymbol{a}\rangle\langle\boldsymbol{b}\rangle\rangle_{\mu} & :=(-1)^{\sum_{k=1}^{s}(\mu(k)-k)}\left\langle a_{\lfloor 1 ; \mu\rfloor} b_{1-\lfloor 1 ; \mu\rfloor}, \ldots, a_{\lfloor s+t ; \mu\rfloor} b_{s+t-\lfloor s+t ; \mu\rfloor}\right\rangle \text { and } \\
\langle\langle\boldsymbol{a}\rangle\langle\boldsymbol{b}\rangle\rangle & :=\sum_{\mu \in M(s, s+t)}\langle\langle\boldsymbol{a}\rangle\langle\boldsymbol{b}\rangle\rangle_{\mu},
\end{aligned}
$$

where $a_{0}=b_{0}=e_{\lambda}$. For example, we have

$$
\begin{aligned}
\left\langle\left\langle a_{1}, a_{2}\right\rangle\left\langle b_{1}, b_{2}\right\rangle\right\rangle= & \left\langle a_{1}, a_{2}, a_{2} b_{1}, a_{2} b_{2}\right\rangle-\left\langle a_{1}, a_{1} b_{1}, a_{2} b_{1}, a_{2} b_{2}\right\rangle \\
& +\left\langle b_{1}, a_{1} b_{1}, a_{2} b_{1}, a_{2} b_{2}\right\rangle+\left\langle a_{1}, a_{1} b_{1}, a_{1} b_{2}, a_{2} b_{2}\right\rangle \\
& -\left\langle b_{1}, a_{1} b_{1}, a_{1} b_{2}, a_{2} b_{2}\right\rangle+\left\langle b_{1}, b_{2}, a_{1} b_{2}, a_{2} b_{2}\right\rangle .
\end{aligned}
$$

for elements $a_{1}, a_{2}, b_{1}, b_{2} \in G_{\lambda}$.

Definition 2.10. Let $D_{n}(X ; \mathbb{Z})_{Y}$ be the submodule of $P_{n}(X ; \mathbb{Z})_{Y}$ generated by elements of the form $\langle y\rangle\left\langle\boldsymbol{a}_{1}\right\rangle \cdots\langle\boldsymbol{a}\rangle\langle\boldsymbol{b}\rangle \cdots\left\langle\boldsymbol{a}_{k}\right\rangle-\langle y\rangle\left\langle\boldsymbol{a}_{1}\right\rangle \cdots\langle\langle\boldsymbol{a}\rangle\langle\boldsymbol{b}\rangle\rangle \cdots\left\langle\boldsymbol{a}_{k}\right\rangle$. When $n \leq 1$, we set $D_{n}(X ; \mathbb{Z})_{Y}=0$.

For example, the submodule $D_{2}(X ; \mathbb{Z})_{Y}$ is generated by the elements of the form

$$
\langle y\rangle\langle a\rangle\langle b\rangle-\langle y\rangle\langle a, a b\rangle+\langle y\rangle\langle b, a b\rangle,
$$

for $y \in Y, a, b \in G_{\lambda}$. 
Proposition $2.11\left([14) . D_{*}(X ; \mathbb{Z})_{Y}:=\left(D_{n}(X ; \mathbb{Z})_{Y}, \partial_{n}\right)_{n \in \mathbb{Z}}\right.$ is a subcomplex of $P_{*}(X ; \mathbb{Z})_{Y}$.

We have a chain complex $C_{*}(X ; \mathbb{Z})_{Y}:=P_{*}(X ; \mathbb{Z})_{Y} / D_{*}(X ; \mathbb{Z})_{Y}$. The homology group $H_{n}(X ; \mathbb{Z})_{Y}$ and the cohomology group $H^{n}(X ; \mathbb{Z})_{Y}$ of the multiple conjugation biquandle $X$ with $X$-set $Y$ are the homology and cohomology groups of the chain complex $C_{*}(X ; \mathbb{Z})_{Y}$.

For an abelian group $A$, the chain and cochain complexes $C_{*}(X ; A)_{Y}$ and $C^{*}(X ; A)_{Y}$ are also defined in the ordinary way. We denote by $H_{n}(X ; A)_{Y}$ and $H^{n}(X ; A)_{Y}$ its $n$-th homology and cohomology groups respectively. A cocycle of $C^{*}(X ; A)_{Y}$ is called a cocycle with $Y$ of the multiple conjugation biquandle $X$ with $A$. We omit $Y$, that is, we write $C_{*}(X ; \mathbb{Z}):=C_{*}(X ; \mathbb{Z})_{Y}$ if $Y$ is the trivial $X$-set.

\subsection{The (co)homology of groups}

In this subsection, we review chain complexes for groups.

We review the group (co)homology. Let $G$ be a group and let $M$ be a right $\mathbb{Z}[G]$-module, where $\mathbb{Z}[G]$ is the group ring of $G$ over the $\operatorname{ring} \mathbb{Z}$. Let $C_{n}^{\operatorname{gp}}(M ; \mathbb{Z})$ be a free abelian group generated by any element $\left(m_{1}, \ldots, m_{n}\right) \in M^{n}$ if $n \geq 1$, and $C_{n}^{\mathrm{gp}}(M ; \mathbb{Z})=0$ otherwise.

We define the boundary map $\partial_{n}^{\mathrm{gp}}: C_{n}^{\mathrm{gp}}(M ; \mathbb{Z}) \rightarrow C_{n-1}^{\mathrm{gp}}(M ; \mathbb{Z})$ by

$$
\begin{aligned}
\partial_{n}^{\mathrm{gp}}\left(m_{1}, \ldots, m_{n}\right): & =\left(m_{2}, \ldots, m_{n}\right) \\
& +\sum_{i=1}^{n-1}(-1)^{i}\left(m_{1}, \ldots, m_{i-1}, m_{i}+m_{i+1}, m_{i+2}, \ldots, m_{n}\right) \\
& +(-1)^{n}\left(m_{1}, \ldots, m_{n-1}\right)
\end{aligned}
$$

if $n \geq 2$, and $\partial_{n}^{\mathrm{gp}}=0$ otherwise. For example,

$$
\begin{aligned}
\partial_{4}^{\mathrm{gp}}\left(m_{1}, m_{2}, m_{3}, m_{4}\right)= & \left(m_{2}, m_{3}, m_{4}\right)-\left(m_{1}+m_{2}, m_{3}, m_{4}\right)+\left(m_{1}, m_{2}+m_{3}, m_{4}\right) \\
& -\left(m_{1}, m_{2}, m_{3}+m_{4}\right)+\left(m_{1}, m_{2}, m_{3}\right) .
\end{aligned}
$$

Let

$$
C_{n}^{\mathrm{gp}}(M ; \mathbb{Z})_{G}:=C_{n}^{\mathrm{gp}}(M ; \mathbb{Z}) \otimes_{\mathbb{Z}[G]} \mathbb{Z},
$$

where $G$ acts on $\mathbb{Z}$ trivially from the left. In other words, under the diagonal action of $G$ on $C_{n}^{\mathrm{gp}}(M ; \mathbb{Z}), C_{n}^{\mathrm{gp}}(M ; \mathbb{Z})_{G}$ is the $G$-coinvariant part. We note that $\left(m_{1} g, \ldots, m_{n} g\right)=\left(m_{1}, \ldots, m_{n}\right)$ in $C_{n}^{\mathrm{gp}}(M ; \mathbb{Z})_{G}$ for any $\left(m_{1}, \ldots, m_{n}\right) \in M^{n}$ and any $g \in G$. We have the induced boundary map $\partial_{n}^{\mathrm{gp}}: C_{n}^{\mathrm{gp}}(M ; \mathbb{Z})_{G} \rightarrow$ $C_{n-1}^{\mathrm{gp}}(M ; \mathbb{Z})_{G}$.

Proposition $2.12([9]) . C_{*}^{\mathrm{gp}}(M ; \mathbb{Z})_{G}=\left(C_{n}^{\mathrm{gp}}(M ; \mathbb{Z})_{G}, \partial_{n}^{\mathrm{gp}}\right)_{n \in \mathbb{Z}}$ is a chain complex.

The chain complex determines the homology group $H_{n}^{\mathrm{gp}}(M ; \mathbb{Z})_{G}$ and the cohomology group $H_{\text {gp }}^{n}(M ; \mathbb{Z})^{G}$. For an abelian group $A$, the chain and cochain complexes $C_{*}^{\mathrm{gp}}(M ; A)_{G}$ and $C_{\mathrm{gp}}^{*}(M ; A)^{G}$ are also defined in the ordinary way. We denote by $H_{n}^{\mathrm{gp}}(M ; A)_{G}$ and $H_{\mathrm{gp}}^{n}(M ; A)^{G}$ its homology and cohomology groups respectively. 
Let $D_{n}^{\mathrm{gp}}(M ; \mathbb{Z})_{G}$ be the submodule of $C_{n}^{\mathrm{gp}}(M ; \mathbb{Z})_{G}$ generated by the elements of the following set

$$
\bigcup_{i=1}^{n-1}\left\{\left(m_{1}, \ldots, m_{i-1}, 0, m_{i+1}, \ldots m_{n}\right) \mid m_{1}, \ldots, m_{n} \in M\right\}
$$

for $n \geq 2$. Define $D_{n}^{\mathrm{gp}}(M ; \mathbb{Z})_{G}=0$ for $n \leq 1$.

Proposition $2.13\left([9) . D_{*}^{\mathrm{gp}}(M ; \mathbb{Z})_{G}:=\left(D_{n}^{\mathrm{gp}}(M ; \mathbb{Z})_{G}, \partial_{n}^{\mathrm{gp}}\right)_{n \in \mathbb{Z}}\right.$ is a subcomplex of $C_{*}^{\mathrm{gp}}(M ; \mathbb{Z})_{G}$.

The chain complex $C_{*}^{\text {norgp }}(M ; \mathbb{Z})_{G}:=C_{*}^{\mathrm{gp}}(M ; \mathbb{Z})_{G} / D_{*}^{\mathrm{gp}}(M ; \mathbb{Z})_{G}$ determines the homology group $H_{n}^{\text {norgp }}(M ; \mathbb{Z})_{G}$ called the normalized group homology. In the ordinary way, for an abelian group $A$, the homology group $H_{n}^{\text {norgp }}(M ; A)_{G}$ and the cohomology group $H_{\text {norgp }}^{n}(M ; A)_{G}$ are defined.

\section{Cocycles of $G$-Alexander biquandles with triv- ial $X$-set}

Throughout this section, let $X=M \times G$ be the $G$-Alexander biquandle of $(M, \varphi)$, see Definition 2.5. We suppose that $X$ has trivial $X$-set.

We define a chain complex $C_{*}^{\mathrm{BR}_{\mathrm{U}}}(X ; \mathbb{Z})$ and chain maps $\gamma$ and $\psi$. It turns out that the chain complex $C_{*}^{\mathrm{BR}_{\mathrm{U}}^{*}}(X ; \mathbb{Z})$ is isomorphic to the birack chain complex $C_{*}^{\mathrm{BR}}(X ; \mathbb{Z})$, and hence the homology group $H_{n}^{\mathrm{BR}}(X ; \mathbb{Z})$ is isomorphic to $H_{n}^{\mathrm{BR}}(X ; \mathbb{Z})$. Through this chain complex, we associate cocycles of the chain complex of group $G$ to cocycles of the birack chain complex.

Our goal in this section is to give Theorems 3.4 and 3.5 .

\subsection{The chain complex $C_{*}^{\mathrm{BR}_{\mathrm{U}}}(X ; \mathbb{Z})$ and chain maps $\gamma$ and $\psi$}

3.1.1 The chain complex $C_{*}^{\mathrm{BR}_{\mathrm{U}}}(X ; \mathbb{Z})$ and the homology group $H_{n}^{\mathrm{BR}}(X ; \mathbb{Z})$

For $\boldsymbol{g}=\left(g_{1}, \ldots, g_{n}\right) \in G^{n}$ and $\boldsymbol{m}=\left(m_{1}, \ldots, m_{n}\right) \in M^{n}$, we use the following notations:

$$
\begin{aligned}
& \boldsymbol{g}_{\{i\}}:=\left(g_{1}, \ldots, g_{i}, g_{i+2}, \ldots, g_{n}\right) \in G^{n-1}, \\
& \boldsymbol{g}_{\{\triangleleft i\}}:=\left(g_{i+1}^{-1} g_{1} g_{i+1}, \ldots, g_{i+1}^{-1} g_{i} g_{i+1}, g_{i+2}, \ldots, g_{n}\right) \in G^{n-1}, \\
& \boldsymbol{m}_{\{i\}}:=\left(m_{1}, \ldots, m_{i-1}, m_{i}+m_{i+1}, m_{i+2}, \ldots, m_{n}\right) \in M^{n-1} \text { and } \\
& \boldsymbol{m}_{\{\triangleleft i\}}:=\left(m_{1} g_{i+1}, \ldots, m_{i-1} g_{i+1}, m_{i} g_{i+1}+m_{i+1} \varphi\left(g_{i+1}\right),\right. \\
&\left.m_{i+2} \varphi\left(g_{i+1}\right), \ldots, m_{n} \varphi\left(g_{i+1}\right)\right) \in M^{n-1},
\end{aligned}
$$

where $n$ and $i$ are integers such that $n \geq 2$ and $0 \leq i \leq n-1$.

Let $C_{n}^{\mathrm{BR}_{\mathrm{U}}}(X ; \mathbb{Z})$ be the free abelian group generated by the elements $(\boldsymbol{g} ; \boldsymbol{m})=$ $\left(g_{1}, \ldots, g_{n} ; m_{1}, \ldots, m_{n}\right) \in G^{n} \times M^{n}$ if $n \geq 1$, and $C_{n}^{\mathrm{BR}}(X ; \mathbb{Z})=0$ otherwise. Define a boundary map $\partial_{n}^{\mathrm{BR}_{\mathrm{U}}}: C_{n}^{\mathrm{BR}_{\mathrm{U}}}(X ; \mathbb{Z}) \rightarrow C_{n-1}^{\mathrm{BR}}(X ; \mathbb{Z})$ by

$$
\partial_{n}^{\mathrm{BR}}(\boldsymbol{g} ; \boldsymbol{m})=\sum_{i=0}^{n-1}(-1)^{i}\left\{\left(\boldsymbol{g}_{\{i\}} ; \boldsymbol{m}_{\{i\}}\right)-\left(\boldsymbol{g}_{\{\triangleleft i\}} ; \boldsymbol{m}_{\{\triangleleft i\}}\right)\right\}
$$


for $n \geq 2$, and define $\partial_{n}^{\mathrm{BR}_{\mathrm{U}}}=0$ for $n \leq 1$. For example, we have

$$
\begin{aligned}
\partial_{3}^{\mathrm{BR}} & \left(g_{1}, g_{2}, g_{3} ; m_{1}, m_{2}, m_{3}\right) \\
= & \left\{\left(g_{2}, g_{3} ; m_{2}, m_{3}\right)-\left(g_{2}, g_{3} ; m_{2} \varphi\left(g_{1}\right), m_{3} \varphi\left(g_{1}\right)\right)\right\} \\
& -\left\{\left(g_{1}, g_{3} ; m_{1}+m_{2}, m_{3}\right)-\left(g_{2}^{-1} g_{1} g_{2}, g_{3} ; m_{1} g_{2}+m_{2} \varphi\left(g_{2}\right), m_{3} \varphi\left(g_{2}\right)\right)\right\} \\
& +\left\{\left(g_{1}, g_{2} ; m_{1}, m_{2}+m_{3}\right)-\left(g_{3}^{-1} g_{1} g_{3}, g_{3}^{-1} g_{2} g_{3} ; m_{1} g_{3}, m_{2} g_{3}+m_{3} \varphi\left(g_{3}\right)\right)\right\} .
\end{aligned}
$$

Lemma 3.1. $C_{*}^{\mathrm{BR}_{\mathrm{U}}}(X ; \mathbb{Z}):=\left(C_{n}^{\mathrm{BR}_{\mathrm{U}}}(X ; \mathbb{Z}), \partial_{n}^{\mathrm{BR}_{\mathrm{U}}}\right)_{n \in \mathbb{Z}}$ is a chain complex.

Proof. We fix an integer $n$ with $n \geq 2$. Define $\partial_{n}^{i}: C_{n}^{\mathrm{BR}_{\mathrm{U}}}(X ; \mathbb{Z}) \rightarrow C_{n-1}^{\mathrm{BR}}(X ; \mathbb{Z})$ by $\partial_{n}^{i}(\boldsymbol{g} ; \boldsymbol{m}):=\left(\boldsymbol{g}_{\{i\}} ; \boldsymbol{m}_{\{i\}}\right)$ and $\delta_{n}^{i}: C_{n}^{\mathrm{BR}_{\mathrm{U}}}(X ; \mathbb{Z}) \rightarrow C_{n-1}^{\mathrm{BR}}(X ; \mathbb{Z})$ by $\delta_{n}^{i}(\boldsymbol{g} ; \boldsymbol{m}):=$ $\left(\boldsymbol{g}_{\{\triangleleft i\}} ; \boldsymbol{m}_{\{\triangleleft i\}}\right)$ for any integer $i$ with $0 \leq i \leq n-1$. Then, we have

$$
\partial_{n}^{\mathrm{BR}}=\sum_{i=0}^{n-1}(-1)^{i}\left(\partial_{n}^{i}-\delta_{n}^{i}\right) .
$$

We assert that

$$
\begin{aligned}
& \delta_{n-1}^{j} \circ \delta_{n}^{i}=\delta_{n-1}^{i} \circ \delta_{n}^{j+1}, \\
& \partial_{n-1}^{j} \circ \partial_{n}^{i}=\partial_{n-1}^{i} \circ \partial_{n}^{j+1}, \\
& \partial_{n-1}^{j} \circ \delta_{n}^{i}=\delta_{n-1}^{i} \circ \partial_{n}^{j+1} \text { and } \\
& \delta_{n-1}^{j} \circ \partial_{n}^{i}=\partial_{n-1}^{i} \circ \delta_{n}^{j+1} .
\end{aligned}
$$

We show the equality (7). Since the other equalities are shown in a similar way, we omit the proofs.

Let $i$ and $j$ be integers with $0 \leq i \leq j \leq n-2$. We see $\boldsymbol{g}_{\{\triangleleft i\}\{\triangleleft j\}}=$ $\boldsymbol{g}_{\{\triangleleft(j+1)\}\{\triangleleft i\}}$ as follows:

$$
\begin{aligned}
\boldsymbol{g}_{\{\triangleleft i\}\{\triangleleft j\}}= & \left(g_{j+2}^{-1} g_{i+1}^{-1} g_{1} g_{i+1} g_{j+2}, \ldots, g_{j+2}^{-1} g_{i+1}^{-1} g_{i} g_{i+1} g_{j+2}, g_{j+2}^{-1} g_{i+2} g_{j+2}, \ldots,\right. \\
& \left.g_{j+2}^{-1} g_{j+1} g_{j+2}, g_{j+3}, \ldots, g_{n}\right) \\
= & \left(\left(g_{j+2}^{-1} g_{i+1} g_{j+2}\right)^{-1} g_{j+2}^{-1} g_{1} g_{j+2}\left(g_{j+2}^{-1} g_{i+1} g_{j+2}\right), \ldots,\right. \\
& \left(g_{j+2}^{-1} g_{i+1} g_{j+2}\right)^{-1} g_{j+2}^{-1} g_{i} g_{j+2}\left(g_{j+2}^{-1} g_{i+1} g_{j+2}\right), g_{j+2}^{-1} g_{i+2} g_{j+2}, \ldots, \\
& \left.g_{j+2}^{-1} g_{j+1} g_{j+2}, g_{j+3}, \ldots, g_{n}\right) \\
= & \boldsymbol{g}_{\{\triangleleft(j+1)\}\{\triangleleft i\}} .
\end{aligned}
$$

We see $\delta_{n-1}^{j} \circ \delta_{n}^{i}=\delta_{n-1}^{i} \circ \delta_{n}^{j+1}$ for $0<i<j \leq n-2$ as follows:

$$
\begin{aligned}
& \delta_{n-1}^{j} \circ \delta_{n}^{i}(\boldsymbol{g} ; \boldsymbol{m}) \\
&=\left(\boldsymbol{g}_{\{\triangleleft i\}\{\triangleleft j\}} ; m_{1} g_{i+1} g_{j+2}, \ldots, m_{i-1} g_{i+1} g_{j+2}, m_{i} g_{i+1} g_{j+2}+m_{i+1} \varphi\left(g_{i+1}\right) g_{j+2},\right. \\
& m_{i+2} \varphi\left(g_{i+1}\right) g_{j+2}, \ldots, m_{j} \varphi\left(g_{i+1}\right) g_{j+2}, m_{j+1} \varphi\left(g_{i+1}\right) g_{j+2}+m_{j+2} \varphi\left(g_{i+1}\right) \varphi\left(g_{j+2}\right), \\
&\left.m_{j+3} \varphi\left(g_{i+1}\right) \varphi\left(g_{j+2}\right), \ldots, m_{n} \varphi\left(g_{i+1}\right) \varphi\left(g_{j+2}\right)\right) \\
&=\left(\boldsymbol{g}_{\{\triangleleft(j+1)\}\{\triangleleft i\}} ; m_{1} g_{j+2}\left(g_{j+2}^{-1} g_{i+1} g_{j+2}\right), \ldots, m_{i-1} g_{j+2}\left(g_{j+2}^{-1} g_{i+1} g_{j+2}\right),\right. \\
& m_{i} g_{j+2}\left(g_{j+2}^{-1} g_{i+1} g_{j+2}\right)+m_{i+1} g_{j+2} \varphi\left(g_{j+2}^{-1} g_{i+1} g_{j+2}\right), m_{i+2} g_{j+2} \varphi\left(g_{j+2}^{-1} g_{i+1} g_{j+2}\right), \ldots, \\
& m_{j} g_{j+2} \varphi\left(g_{j+2}^{-1} g_{i+1} g_{j+2}\right), m_{j+1} g_{j+2} \varphi\left(g_{j+2}^{-1} g_{i+1} g_{j+2}\right)+m_{j+2} \varphi\left(g_{j+2}\right) \varphi\left(g_{j+2}^{-1} g_{i+1} g_{j+2}\right), \\
&\left.m_{j+3} \varphi\left(g_{j+2}\right) \varphi\left(g_{j+2}^{-1} g_{i+1} g_{j+2}\right), \ldots, m_{n} \varphi\left(g_{j+2}\right) \varphi\left(g_{j+2}^{-1} g_{i+1} g_{j+2}\right)\right) \\
&= \delta_{n-1}^{i} \circ \delta_{n}^{j+1}(\boldsymbol{g} ; \boldsymbol{m}) .
\end{aligned}
$$


For the cases that $i=j$ or $i=0$, we can show that the equality (7) holds.

Hence, we have the equality (7) for any $i, j$ with $0 \leq i \leq j \leq n-2$.

By using the equalities (17)-(10), we see that $\partial_{n-1}^{\mathrm{BR}} \circ \partial_{n}^{\mathrm{BR}}=0$.

The $n$-th homology and cohomology groups of the chain complex $C_{*}^{\mathrm{BR}}(X ; \mathbb{Z})$ are denoted by $H_{n}^{\mathrm{BR}}(X ; \mathbb{Z})$ and $H_{\mathrm{BR}_{\mathrm{U}}}^{n}(X ; \mathbb{Z})$ respectively.

\subsubsection{The chain map $\gamma: C_{*}^{\mathrm{BR}}(X ; \mathbb{Z}) \rightarrow C_{*}^{\mathrm{BR}}(X ; \mathbb{Z})$}

For $n \geq 1$, define a homomorphism $\gamma_{n}: C_{n}^{\mathrm{BR}}(X ; \mathbb{Z}) \rightarrow C_{n}^{\mathrm{BR}}(X ; \mathbb{Z})$ by

$$
\gamma_{n}\left(\left(m_{1}, g_{1}\right), \ldots,\left(m_{n}, g_{n}\right)\right):=\left(\boldsymbol{g} ; m_{1}^{\prime}, \ldots, m_{n-1}^{\prime}, m_{n}\right),
$$

where we write $m_{i}^{\prime}:=m_{i}-m_{i+1}$ and $\boldsymbol{g}=\left(g_{1}, \ldots, g_{n}\right)$. For $n<1$, define $\gamma_{n}=0$.

Lemma 3.2. (1) The map $\gamma$ is a chain map, that is, it holds that

$$
\gamma_{n-1} \circ \partial_{n}^{\mathrm{BR}}=\partial_{n}^{\mathrm{BR}_{\mathrm{U}}} \circ \gamma_{n} .
$$

(2) For any integer $n$, the map $\gamma_{n}$ is an isomorphism.

Proof. The assertion that $\gamma$ is a chain map is easily verified by a direct calculation, which is left to the reader. Define a homomorphism $r_{n}: C_{n}^{\mathrm{BR}_{\mathrm{U}}}(X ; \mathbb{Z}) \rightarrow$ $C_{n}^{\mathrm{BR}}(X ; \mathbb{Z})$ by

$$
r_{n}(\boldsymbol{g} ; \boldsymbol{m}):=\left(\left(\sum_{i=1}^{n} m_{i}, g_{1}\right),\left(\sum_{i=2}^{n} m_{i}, g_{2}\right), \ldots,\left(\sum_{i=n}^{n} m_{i}, g_{n}\right)\right) .
$$

We can easily see that $r_{n}$ is the inverse map of $\gamma_{n}$, and thus, $\gamma_{n}$ is a bijection.

\subsubsection{The chain map $\psi: C_{*}^{\mathrm{BR}}(X ; \mathbb{Z}) \rightarrow C_{*}^{\mathrm{gp}}(M ; \mathbb{Z})_{G}$}

For $n \geq 1$, define $\psi_{n}: C_{n}^{\mathrm{BR}}(X ; \mathbb{Z}) \rightarrow C_{n}^{\mathrm{gp}}(M ; \mathbb{Z})_{G}$ by

$$
\psi_{n}(\boldsymbol{g} ; \boldsymbol{m}):=\sum_{\boldsymbol{k}=\left(k_{1}, \ldots, k_{n}\right) \in \mathcal{K}_{n}}(-1)^{|\boldsymbol{k}|}\left(m_{1} \boldsymbol{g}_{\boldsymbol{k}_{1}}, m_{2} \boldsymbol{g}_{\boldsymbol{k}_{2}}, \ldots, m_{n} \boldsymbol{g}_{\boldsymbol{k}_{n}}\right),
$$

where $\mathcal{K}_{n}:=\left\{\boldsymbol{k}=\left(k_{1}, \ldots, k_{n}\right) \in\{0,1\}^{n} \mid k_{1}=0\right\}$ and

$$
\begin{aligned}
|\boldsymbol{k}| & :=k_{1}+k_{2}+\cdots+k_{n} \text { and } \\
\boldsymbol{g}_{\boldsymbol{k}_{i}} & :=\varphi\left(g_{1}^{k_{1}} g_{2}^{k_{2}} \cdots g_{i}^{k_{i}}\right) g_{i+1}^{k_{i+1}} g_{i+2}^{k_{i+2}} \cdots g_{n}^{k_{n}} \in G
\end{aligned}
$$

for an element $\boldsymbol{k}=\left(k_{1}, \ldots, k_{n}\right) \in \mathcal{K}_{n}$ and an integer $i$ with $1 \leq i \leq n$. For $n<1$, define $\psi_{n}=0$.

For example, we have

$$
\begin{aligned}
\psi_{3}(\boldsymbol{g} ; \boldsymbol{m})= & (-1)^{0+1+1}\left(m_{1} \varphi\left(g_{1}^{0}\right) g_{2}^{1} g_{3}^{1}, m_{2} \varphi\left(g_{1}^{0} g_{2}^{1}\right) g_{3}^{1}, m_{3} \varphi\left(g_{1}^{0} g_{2}^{1} g_{3}^{1}\right)\right) \\
+ & (-1)^{0+1+0}\left(m_{1} \varphi\left(g_{1}^{0}\right) g_{2}^{1} g_{3}^{0}, m_{2} \varphi\left(g_{1}^{0} g_{2}^{1}\right) g_{3}^{0}, m_{3} \varphi\left(g_{1}^{0} g_{2}^{1} g_{3}^{0}\right)\right) \\
+ & (-1)^{0+0+1}\left(m_{1} \varphi\left(g_{1}^{0}\right) g_{2}^{0} g_{3}^{1}, m_{2} \varphi\left(g_{1}^{0} g_{2}^{0}\right) g_{3}^{1}, m_{3} \varphi\left(g_{1}^{0} g_{2}^{0} g_{3}^{1}\right)\right) \\
+ & (-1)^{0+0+0}\left(m_{1} \varphi\left(g_{1}^{0}\right) g_{2}^{0} g_{3}^{0}, m_{2} \varphi\left(g_{1}^{0} g_{2}^{0}\right) g_{3}^{0}, m_{3} \varphi\left(g_{1}^{0} g_{2}^{0} g_{3}^{0}\right)\right) \\
= & \left(m_{1} g_{2} g_{3}, m_{2} \varphi\left(g_{2}\right) g_{3}, m_{3} \varphi\left(g_{2} g_{3}\right)\right) \\
& -\left(m_{1} g_{2}, m_{2} \varphi\left(g_{2}\right), m_{3} \varphi\left(g_{2}\right)\right) \\
- & \left(m_{1} g_{3}, m_{2} g_{3}, m_{3} \varphi\left(g_{3}\right)\right) \\
+ & \left(m_{1}, m_{2}, m_{3}\right) .
\end{aligned}
$$


Lemma 3.3. The map $\psi$ is a chain map, that is, it holds that

$$
\psi_{n-1} \circ \partial_{n}^{\mathrm{BR}}=\partial_{n}^{\mathrm{gp}} \circ \psi_{n} .
$$

Proof. We fix an integer $n \geq 2$, and fix $\boldsymbol{g}=\left(g_{1}, \ldots, g_{n}\right)$ and $\boldsymbol{m}=\left(m_{1}, \ldots, m_{n}\right)$.

For any integer $i$ with $2 \leq i \leq n$, let

$$
\begin{aligned}
& \mathcal{K}_{i}^{0}:=\left\{\boldsymbol{k}=\left(k_{1}, \ldots, k_{n}\right) \in\{0,1\}^{n} \mid k_{1}=k_{i}=0\right\} \text { and } \\
& \mathcal{K}_{i}^{1}:=\left\{\boldsymbol{k}=\left(k_{1}, \ldots, k_{n}\right) \in\{0,1\}^{n} \mid k_{1}=0, k_{i}=1\right\}
\end{aligned}
$$

For any $i$ with $1 \leq i \leq n-1$, put

$$
\begin{aligned}
A_{i} & :=\left(m_{1} \boldsymbol{g}_{\boldsymbol{k}_{1}}, \ldots, m_{i-1} \boldsymbol{g}_{\boldsymbol{k}_{i-1}}, m_{i} \boldsymbol{g}_{\boldsymbol{k}_{i}}+m_{i+1} \boldsymbol{g}_{\boldsymbol{k}_{i+1}}, m_{i+2} \boldsymbol{g}_{\boldsymbol{k}_{i+2}}, \ldots, m_{n} \boldsymbol{g}_{\boldsymbol{k}_{n}}\right) \\
L & :=\sum_{\boldsymbol{k} \in \mathcal{K}_{n}}(-1)^{|\boldsymbol{k}|} \sum_{i=1}^{n-1}(-1)^{i} A_{i}
\end{aligned}
$$

We show that $\psi_{n-1} \circ \partial_{n}^{\mathrm{BR}}(\boldsymbol{g} ; \boldsymbol{m})=L=\partial_{n}^{\mathrm{gp}} \circ \psi_{n}(\boldsymbol{g} ; \boldsymbol{m})$.

Firstly, we show $\psi_{n-1} \circ \partial_{n}^{\mathrm{BR}_{\mathrm{U}}}(\boldsymbol{g} ; \boldsymbol{m})=L$. We have

$$
\begin{aligned}
\psi_{n-1} \circ \partial_{n}^{\mathrm{BR}}(\boldsymbol{g} ; \boldsymbol{m})= & \psi_{n-1}\left(\left(\boldsymbol{g}_{\{0\}} ; \boldsymbol{m}_{\{0\}}\right)-\left(\boldsymbol{g}_{\{\triangleleft 0\}} ; \boldsymbol{m}_{\{\triangleleft 0\}}\right)\right) \\
& +\sum_{i=1}^{n-1}(-1)^{i} \psi_{n-1}\left(\left(\boldsymbol{g}_{\{i\}} ; \boldsymbol{m}_{\{i\}}\right)-\left(\boldsymbol{g}_{\{\triangleleft i\}} ; \boldsymbol{m}_{\{\triangleleft i\}}\right)\right) .
\end{aligned}
$$

Since $\left(m_{1} g, \ldots, m_{n} g\right)=\left(m_{1}, \ldots, m_{n}\right)$ in $C_{n}^{\mathrm{gp}}(M ; \mathbb{Z})_{G}$ for any $\left(m_{1}, \ldots, m_{n}\right) \in$ $M^{n}$ and any $g \in G$, we see that

$$
\begin{aligned}
& \psi_{n-1}\left(\boldsymbol{g}_{\{0\}} ; \boldsymbol{m}_{\{0\}}\right)-\psi_{n-1}\left(\boldsymbol{g}_{\{\triangleleft 0\}} ; \boldsymbol{m}_{\{\triangleleft 0\}}\right) \\
& =\sum_{\boldsymbol{k} \in \mathcal{K}_{2}^{0}}(-1)^{|\boldsymbol{k}|}\left\{\left(m_{2} \boldsymbol{g}_{\boldsymbol{k}_{2}}, \ldots, m_{n} \boldsymbol{g}_{\boldsymbol{k}_{n}}\right)-\left(m_{2} \varphi\left(g_{1}\right) \boldsymbol{g}_{\boldsymbol{k}_{2}}, \ldots, m_{n} \varphi\left(g_{1}\right) \boldsymbol{g}_{\boldsymbol{k}_{n}}\right)\right\} \\
& =\sum_{\boldsymbol{k} \in \mathcal{K}_{2}^{0}}(-1)^{|\boldsymbol{k}|}\left\{\left(m_{2} \boldsymbol{g}_{\boldsymbol{k}_{2}}, \ldots, m_{n} \boldsymbol{g}_{\boldsymbol{k}_{n}}\right)-\left(m_{2} \boldsymbol{g}_{\boldsymbol{k}_{2}} \varphi\left(g_{1}\right), \ldots, m_{n} \boldsymbol{g}_{\boldsymbol{k}_{n}} \varphi\left(g_{1}\right)\right)\right\} \\
& =0 .
\end{aligned}
$$

For any integer $i$ with $1 \leq i \leq n-1$, we have

$$
\begin{aligned}
& \psi_{n-1}\left(\boldsymbol{g}_{\{i\}} ; \boldsymbol{m}_{\{i\}}\right) \\
& =\sum_{\boldsymbol{k} \in \mathcal{K}_{i+1}^{0}}(-1)^{|\boldsymbol{k}|}( \\
& \quad m_{1} \varphi\left(g_{1}^{k_{1}}\right) g_{2}^{k_{2}} \cdots g_{i}^{k_{i}} g_{i+1}^{0} g_{i+2}^{k_{i+2}} \cdots g_{n}^{k_{n}}, \ldots, \\
& \quad m_{i-1} \varphi\left(g_{1}^{k_{1}} \cdots g_{i-1}^{k_{i-1}}\right) g_{i}^{k_{i}} g_{i+1}^{0} g_{i+2}^{k_{i+2}} \cdots g_{n}^{k_{n}}, \\
& \quad m_{i} \varphi\left(g_{1}^{k_{1}} \cdots g_{i}^{k_{i}}\right) g_{i+1}^{0} g_{i+2}^{k_{i+2}} \cdots g_{n}^{k_{n}}+m_{i+1} \varphi\left(g_{1}^{k_{1}} \cdots g_{i}^{k_{i}} g_{i+1}^{0}\right) g_{i+2}^{k_{i+2}} \cdots g_{n}^{k_{n}}, \\
& \quad m_{i+2} \varphi\left(g_{1}^{k_{1}} \cdots g_{i}^{k_{i}} g_{i+1}^{0} g_{i+2}^{k_{i+2}}\right) g_{i+3}^{k_{i+3}} \cdots g_{n}^{k_{n}}, \ldots, \\
& \left.\quad m_{n} \varphi\left(g_{1}^{k_{1}} g_{2}^{k_{2}} \cdots g_{i}^{k_{i}} g_{i+1}^{0} g_{i+2}^{k_{i+2}} \cdots g_{n}^{k_{n}}\right)\right) \\
& =\sum_{\boldsymbol{k} \in \mathcal{K}_{i+1}^{0}}(-1)^{|\boldsymbol{k}|} A_{i} .
\end{aligned}
$$


For any integer $i$ with $1 \leq i \leq n-1$, we also have

$$
\begin{aligned}
& \psi_{n-1}\left(\boldsymbol{g}_{\{\triangleleft i\}} ; \boldsymbol{m}_{\{\triangleleft i\}}\right) \\
& =\sum_{\boldsymbol{k} \in \mathcal{K}_{i+1}^{0}}(-1)^{|\boldsymbol{k}|}( \\
& m_{1} g_{i+1} \varphi\left(\left(g_{i+1}^{-1} g_{1} g_{i+1}\right)^{k_{1}}\right)\left(g_{i+1}^{-1} g_{2} g_{i+1}\right)^{k_{2}} \cdots\left(g_{i+1}^{-1} g_{i} g_{i+1}\right)^{k_{i}} g_{i+1}^{0} g_{i+2}^{k_{i+2}} \cdots g_{n}^{k_{n}}, \ldots, \\
& m_{i-1} g_{i+1} \varphi\left(\left(g_{i+1}^{-1} g_{1} g_{i+1}\right)^{k_{1}} \cdots\left(g_{i+1}^{-1} g_{i-1} g_{i+1}\right)^{k_{i-1}}\right)\left(g_{i+1}^{-1} g_{i} g_{i+1}\right)^{k_{i}} g_{i+1}^{0} g_{i+2}^{k_{i+2}} \cdots g_{n}^{k_{n}} \text {, } \\
& m_{i} g_{i+1} \varphi\left(\left(g_{i+1}^{-1} g_{1} g_{i+1}\right)^{k_{1}} \cdots\left(g_{i+1}^{-1} g_{i} g_{i+1}\right)^{k_{i}}\right) g_{i+1}^{0} g_{i+2}^{k_{i+2}} \cdots g_{n}^{k_{n}} \\
& +m_{i+1} \varphi\left(g_{i+1}\right) \varphi\left(\left(g_{i+1}^{-1} g_{1} g_{i+1}\right)^{k_{1}} \cdots\left(g_{i+1}^{-1} g_{i} g_{i+1}\right)^{k_{i}} g_{i+1}^{0}\right) g_{i+2}^{k_{i+2}} \cdots g_{n}^{k_{n}} \text {, } \\
& m_{i+2} \varphi\left(g_{i+1}\right) \varphi\left(\left(g_{i+1}^{-1} g_{1} g_{i+1}\right)^{k_{1}} \cdots\left(g_{i+1}^{-1} g_{i} g_{i+1}\right)^{k_{i}} g_{i+1}^{0} g_{i+2}^{k_{i+2}}\right) g_{i+3}^{k_{i+3}} \cdots g_{n}^{k_{n}}, \ldots, \\
& \left.m_{n} \varphi\left(g_{i+1}\right) \varphi\left(\left(g_{i+1}^{-1} g_{1} g_{i+1}\right)^{k_{1}} \cdots\left(g_{i+1}^{-1} g_{i} g_{i+1}\right)^{k_{i}} g_{i+1}^{0} g_{i+2}^{k_{i+2}} \cdots g_{n}^{k_{n}}\right)\right) \\
& =\sum_{\boldsymbol{k} \in \mathcal{K}_{i+1}^{0}}(-1)^{|\boldsymbol{k}|}( \\
& m_{1} g_{i+1} \varphi\left(g_{1}^{k_{1}}\right) g_{i+1}^{-1} g_{2}^{k_{2}} \cdots g_{i}^{k_{i}} g_{i+1}^{1} g_{i+1}^{0} g_{i+2}^{k_{i+2}} \cdots g_{n}^{k_{n}}, \ldots, \\
& m_{i-1} g_{i+1} \varphi\left(g_{1}^{k_{1}} g_{2}^{k_{2}} \cdots g_{i-1}^{k_{i-1}}\right) g_{i+1}^{-1} g_{i}^{k_{i}} g_{i+1}^{1} g_{i+1}^{0} g_{i+2}^{k_{i+2}} \cdots g_{n}^{k_{n}} \text {, } \\
& m_{i} g_{i+1} \varphi\left(g_{1}^{k_{1}} g_{2}^{k_{2}} \cdots g_{i}^{k_{i}}\right) g_{i+1}^{0} g_{i+2}^{k_{i+2}} \cdots g_{n}^{k_{n}} \\
& +m_{i+1} \varphi\left(g_{i+1}\right) \varphi\left(g_{1}^{k_{1}} g_{2}^{k_{2}} \cdots g_{i}^{k_{i}} g_{i+1}^{0}\right) g_{i+2}^{k_{i+2}} \cdots g_{n}^{k_{n}} \text {, } \\
& m_{i+2} \varphi\left(g_{i+1}\right) \varphi\left(g_{1}^{k_{1}} g_{2}^{k_{2}} \cdots g_{i}^{k_{i}} g_{i+1}^{0} g_{i+2}^{k_{i+2}}\right) g_{i+3}^{k_{i+3}} \cdots g_{n}^{k_{n}}, \ldots \text {, } \\
& \left.m_{n} \varphi\left(g_{i+1}\right) \varphi\left(g_{1}^{k_{1}} g_{2}^{k_{2}} \cdots g_{i}^{k_{i}} g_{i+1}^{0} g_{i+2}^{k_{i+2}} \cdots g_{n}^{k_{n}}\right)\right) \\
& =\sum_{\boldsymbol{k} \in \mathcal{K}_{i+1}^{0}}(-1)^{|\boldsymbol{k}|}( \\
& m_{1} \varphi\left(g_{1}^{k_{1}}\right) g_{2}^{k_{2}} \cdots g_{i}^{k_{i}} g_{i+1}^{1} g_{i+2}^{k_{i+2}} \cdots g_{n}^{k_{n}}, \ldots, \\
& m_{i-1} \varphi\left(g_{1}^{k_{1}} g_{2}^{k_{2}} \cdots g_{i-1}^{k_{i-1}}\right) g_{i}^{k_{i}} g_{i+1}^{1} g_{i+2}^{k_{i+2}} \cdots g_{n}^{k_{n}} \text {, } \\
& m_{i} \varphi\left(g_{1}^{k_{1}} g_{2}^{k_{2}} \cdots g_{i}^{k_{i}}\right) g_{i+1}^{1} g_{i+2}^{k_{i+2}} \cdots g_{n}^{k_{n}}+m_{i+1} \varphi\left(g_{1}^{k_{1}} g_{2}^{k_{2}} \cdots g_{i}^{k_{i}} g_{i+1}^{1}\right) g_{i+2}^{k_{i+2}} \cdots g_{n}^{k_{n}}, \\
& m_{i+2} \varphi\left(g_{1}^{k_{1}} g_{2}^{k_{2}} \cdots g_{i}^{k_{i}} g_{i+1}^{1} g_{i+2}^{k_{i+2}}\right) g_{i+3}^{k_{i+3}} \cdots g_{n}^{k_{n}}, \ldots \text {, } \\
& \left.m_{n} \varphi\left(g_{1}^{k_{1}} g_{2}^{k_{2}} \cdots g_{i}^{k_{i}} g_{i+1}^{1} g_{i+2}^{k_{i+2}} \cdots g_{n}^{k_{n}}\right)\right) \\
& =\sum_{\boldsymbol{k} \in \mathcal{K}_{i+1}^{1}}(-1)^{|\boldsymbol{k}|-1} A_{i}=-\sum_{\boldsymbol{k} \in \mathcal{K}_{i+1}^{1}}(-1)^{|\boldsymbol{k}|} A_{i} \text {. }
\end{aligned}
$$

Using (13) and (14), we have

$$
\begin{aligned}
\psi_{n-1}\left(\left(\boldsymbol{g}_{\{i\}} ; \boldsymbol{m}_{\{i\}}\right)-\left(\boldsymbol{g}_{\{\triangleleft i\}} ; \boldsymbol{m}_{\{\triangleleft i\}}\right)\right) & =\sum_{\boldsymbol{k} \in \mathcal{K}_{i+1}^{0}}(-1)^{|\boldsymbol{k}|} A_{i}+\sum_{\boldsymbol{k} \in \mathcal{K}_{i+1}^{1}}(-1)^{|\boldsymbol{k}|} A_{i} \\
& =\sum_{\boldsymbol{k} \in \mathcal{K}_{n}}(-1)^{|\boldsymbol{k}|} A_{i} .
\end{aligned}
$$

Using (11), (12) and (15), we have

$$
\psi_{n-1} \circ \partial_{n}^{\mathrm{BR}}(\boldsymbol{g} ; \boldsymbol{m})=\sum_{i=1}^{n-1}(-1)^{i} \sum_{\boldsymbol{k} \in \mathcal{K}_{n}}(-1)^{|\boldsymbol{k}|} A_{i}=L .
$$


Secondly, we show $\partial_{n}^{\mathrm{gp}} \circ \psi_{n}(\boldsymbol{g} ; \boldsymbol{m})=L$. We have

$$
\begin{aligned}
\partial_{n}^{\mathrm{gp}} \circ \psi_{n}(\boldsymbol{g} ; \boldsymbol{m})= & \sum_{\boldsymbol{k} \in \mathcal{K}_{n}}(-1)^{|\boldsymbol{k}|} \partial_{n}^{\mathrm{gp}}\left(m_{1} \boldsymbol{g}_{\boldsymbol{k}_{1}}, \ldots, m_{n} \boldsymbol{g}_{\boldsymbol{k}_{n}}\right) \\
= & \sum_{\boldsymbol{k} \in \mathcal{K}_{n}}(-1)^{|\boldsymbol{k}|}\left(m_{2} \boldsymbol{g}_{\boldsymbol{k}_{2}}, \ldots, m_{n} \boldsymbol{g}_{\boldsymbol{k}_{n}}\right)+L \\
& +\sum_{\boldsymbol{k} \in \mathcal{K}_{n}}(-1)^{|\boldsymbol{k}|}(-1)^{n}\left(m_{1} \boldsymbol{g}_{\boldsymbol{k}_{1}}, \ldots, m_{n-1} \boldsymbol{g}_{\boldsymbol{k}_{n-1}}\right) .
\end{aligned}
$$

We have

$$
\begin{aligned}
& \sum_{\boldsymbol{k} \in \mathcal{K}_{n}}(-1)^{|\boldsymbol{k}|}\left(m_{2} \boldsymbol{g}_{\boldsymbol{k}_{2}}, \ldots, m_{n} \boldsymbol{g}_{\boldsymbol{k}_{n}}\right) \\
& =\sum_{\boldsymbol{k} \in \mathcal{K}_{2}^{0}}(-1)^{|\boldsymbol{k}|}\left(m_{2} \boldsymbol{g}_{\boldsymbol{k}_{2}}, \ldots, m_{n} \boldsymbol{g}_{\boldsymbol{k}_{n}}\right)+\sum_{\boldsymbol{k} \in \mathcal{K}_{2}^{1}}(-1)^{|\boldsymbol{k}|}\left(m_{2} \boldsymbol{g}_{\boldsymbol{k}_{2}}, \ldots, m_{n} \boldsymbol{g}_{\boldsymbol{k}_{n}}\right) \\
& =0 .
\end{aligned}
$$

This is because it holds that

$$
\begin{aligned}
& \sum_{\boldsymbol{k} \in \mathcal{K}_{2}^{1}}(-1)^{|\boldsymbol{k}|}\left(m_{2} \boldsymbol{g}_{\boldsymbol{k}_{2}}, \ldots, m_{n} \boldsymbol{g}_{\boldsymbol{k}_{n}}\right) \\
& =\sum_{\boldsymbol{k} \in \mathcal{K}_{2}^{1}}(-1)^{|\boldsymbol{k}|} \quad\left(m_{2} \varphi\left(g_{1}^{k_{1}} g_{2}^{1}\right) g_{3}^{k_{3}} \cdots g_{n}^{k_{n}}, \ldots, m_{n} \varphi\left(g_{1}^{k_{1}} g_{2}^{1} g_{3}^{k_{3}} \cdots g_{n}^{k_{n}}\right)\right) \\
& =\sum_{\boldsymbol{k} \in \mathcal{K}_{2}^{1}}(-1)^{|\boldsymbol{k}|}\left(m_{2} \varphi\left(g_{1}^{k_{1}}\right) g_{3}^{k_{3}} \cdots g_{n}^{k_{n}}, \ldots, m_{n} \varphi\left(g_{1}^{k_{1}} g_{3}^{k_{3}} \cdots g_{n}^{k_{n}}\right)\right) \\
& =\sum_{\boldsymbol{k} \in \mathcal{K}_{2}^{0}}(-1)^{|\boldsymbol{k}|+1}\left(m_{2} \varphi\left(g_{1}^{k_{1}}\right) g_{2}^{0} g_{3}^{k_{3}} \cdots g_{n}^{k_{n}}, \ldots, m_{n} \varphi\left(g_{1}^{k_{1}} g_{2}^{0} g_{3}^{k_{3}} \cdots g_{n}^{k_{n}}\right)\right) \\
& =-\sum_{\boldsymbol{k} \in \mathcal{K}_{2}^{0}}(-1)^{|\boldsymbol{k}|}\left(m_{2} \boldsymbol{g}_{\boldsymbol{k}_{2}}, \ldots, m_{n} \boldsymbol{g}_{\boldsymbol{k}_{n}}\right) .
\end{aligned}
$$

Similarly, we have

$$
\begin{aligned}
& \sum_{\boldsymbol{k} \in \mathcal{K}_{n}}(-1)^{|\boldsymbol{k}|}(-1)^{n}\left(m_{1} \boldsymbol{g}_{\boldsymbol{k}_{1}}, \ldots, m_{n-1} \boldsymbol{g}_{\boldsymbol{k}_{n-1}}\right) \\
& =(-1)^{n}\left(\sum_{\boldsymbol{k} \in \mathcal{K}_{n}^{0}}(-1)^{|\boldsymbol{k}|}\left(m_{1} \boldsymbol{g}_{\boldsymbol{k}_{1}}, \ldots, m_{n-1} \boldsymbol{g}_{\boldsymbol{k}_{n-1}}\right)+\sum_{\boldsymbol{k} \in \mathcal{K}_{n}^{1}}(-1)^{|\boldsymbol{k}|}\left(m_{1} \boldsymbol{g}_{\boldsymbol{k}_{1}}, \ldots, m_{n-1} \boldsymbol{g}_{\boldsymbol{k}_{n-1}}\right)\right) \\
& =0
\end{aligned}
$$

By (16), (17) and (18), we have $\partial_{n}^{\mathrm{gp}} \circ \psi_{n}(\boldsymbol{g} ; \boldsymbol{m})=L$.

\subsection{Birack cocycles of $G$-Alexander biquandles}

Let $\gamma=\left(\gamma_{n}\right)$ and $\psi=\left(\psi_{n}\right)$ be the chain maps defined in Subsection 3.1. We have a sequence

$$
C_{n}^{\mathrm{BR}}(X ; \mathbb{Z}) \stackrel{\gamma_{n}}{\longrightarrow} C_{n}^{\mathrm{BR}}(X ; \mathbb{Z}) \stackrel{\psi_{n}}{\longrightarrow} C_{n}^{\mathrm{gp}}(M ; \mathbb{Z})_{G}
$$

of chain groups $C_{n}^{\mathrm{BR}}(X ; \mathbb{Z}), C_{n}^{\mathrm{BR}}(X ; \mathbb{Z}), C_{n}^{\mathrm{gp}}(M ; \mathbb{Z})_{G}$ and chain maps $\gamma, \psi$ for $n \geq 1$. 
Theorem 3.4. For any $n$-cocycle $f: C_{n}^{\mathrm{gp}}(M ; \mathbb{Z})_{G} \rightarrow A$ of $M$, the map

$$
\Phi_{f}:=f \circ \psi_{n} \circ \gamma_{n}: C_{n}^{\mathrm{BR}}(X ; \mathbb{Z}) \rightarrow A
$$

is a birack $n$-cocycle of the $G$-Alexander biquandle $X$.

Proof. Since $\psi_{n} \circ \gamma_{n}$ is a chain map, we see the result.

An $A$-multilinear map $f: M^{n} \rightarrow A$ is $G$-invariant if $f\left(m_{1} g, \ldots, m_{n} g\right)=$ $f\left(m_{1}, \ldots, m_{n}\right)$ for any $g \in G$ and $\left(m_{1}, \ldots, m_{n}\right) \in M^{n}$. Note that any $G$ invariant $A$-multilinear map $f: M^{n} \rightarrow A$ induces a cocycle $f: C_{n}^{\operatorname{gp}}(M ; \mathbb{Z})_{G} \rightarrow$ A.

The following theorem follows from a direct calculation.

Theorem 3.5. (1) Let $f: M^{2} \rightarrow A$ be a G-invariant A-multilinear map. The birack 2-cocycle $\Phi_{f}=f \circ \psi_{2} \circ \gamma_{2}: C_{2}^{\mathrm{BR}}(X ; \mathbb{Z}) \rightarrow A$ is formulated as

$$
\begin{aligned}
& \Phi_{f}\left(\left(m_{1}, g_{1}\right),\left(m_{2}, g_{2}\right)\right)=f\left(m_{1}-m_{2}, m_{2}\left(1-\varphi\left(g_{2}\right) g_{2}^{-1}\right)\right) \\
& \text { for }\left(\left(m_{1}, g_{1}\right),\left(m_{2}, g_{2}\right)\right) \in X^{2} \subset C_{2}^{\mathrm{BR}}(X ; \mathbb{Z}) .
\end{aligned}
$$

(2) Let $f: M^{3} \rightarrow A$ be a $G$-invariant $A$-multilinear map. The birack 3-cocycle $\Phi_{f}=f \circ \psi_{3} \circ \gamma_{3}: C_{3}^{\mathrm{BR}}(X ; \mathbb{Z}) \rightarrow A$ is formulated as

$$
\begin{aligned}
& \qquad \Phi_{f}\left(\left(m_{1}, g_{1}\right),\left(m_{2}, g_{2}\right),\left(m_{3}, g_{3}\right)\right) \\
& =f\left(\left(m_{1}-m_{2}\right)\left(1-\varphi\left(g_{2}\right)^{-1} g_{2}\right), m_{2}-m_{3}, m_{3}\left(1-\varphi\left(g_{3}\right) g_{3}^{-1}\right)\right) \\
& \text { for }\left(\left(m_{1}, g_{1}\right),\left(m_{2}, g_{2}\right),\left(m_{3}, g_{3}\right)\right) \in X^{3} \subset C_{3}^{\mathrm{BR}}(X ; \mathbb{Z}) .
\end{aligned}
$$

\section{Cocycles of $G$-Alexander biquandles with the $X$-set $X$}

Throughout this section, let $X=M \times G$ be the $G$-Alexander biquandle of $(M, \varphi)$ and we assume that $X$ is also an $X$-set with the action $*:=\underline{*}$, that is, it holds that $\left(x_{0} * x_{1}\right) *\left(x_{2} * x_{1}\right)=\left(x_{0} * x_{2}\right) *\left(x_{1} * x_{2}\right)$ for any $x_{0}, x_{1}, x_{2} \in X$. We discuss birack cocycles of $X$ with the $X$-set $X$.

Since we apply a similar argument as shown in Section 3 to this case, we summarize all the properties without proof.

We define a chain complex of $X=M \times G$ with the $X$-set $X$, denoted by $C_{*}^{\mathrm{BR}_{\mathrm{U}}}(X ; \mathbb{Z})_{X}$, and define chain maps $\gamma$ and $\psi$. It turns out that the chain complex $C_{*}^{\mathrm{BR}}(X ; \mathbb{Z})_{X}$ is isomorphic to the birack chain complex $C_{*}^{\mathrm{BR}}(X ; \mathbb{Z})_{X}$ and hence the homology group $H_{n}^{\mathrm{BR}}(X ; \mathbb{Z})_{X}$ is isomorphic to $H_{n}^{\mathrm{BR}}(X ; \mathbb{Z})_{X}$.

Our goal in this section is to give Theorems 4.4 and 4.5 . 


\subsection{The chain complex $C_{*}^{\mathrm{BR}_{\mathrm{U}}}(X ; \mathbb{Z})_{X}$ and chain maps $\gamma$ and $\psi$}

4.1.1 The chain complex $C_{*}^{\mathrm{BR}_{\mathrm{U}}}(X ; \mathbb{Z})_{X}$ and the homology group $H_{n}^{\mathrm{BR}}(X ; \mathbb{Z})_{X}$

For $\boldsymbol{g}=\left(g_{0}, g_{1}, \ldots, g_{n}\right) \in G \times G^{n}$ and $\boldsymbol{m}=\left(m_{0}, m_{1}, \ldots, m_{n}\right) \in M \times M^{n}$, we use the following notations:

$$
\begin{aligned}
& \boldsymbol{g}_{\{i\}}:=\left(g_{0}, g_{1}, \ldots, g_{i}, g_{i+2}, \ldots, g_{n}\right) \in G \times G^{n-1}, \\
& \boldsymbol{g}_{\{\triangleleft i\}}:=\left(g_{i+1}^{-1} g_{0} g_{i+1}, g_{i+1}^{-1} g_{1} g_{i+1}, \ldots, g_{i+1}^{-1} g_{i} g_{i+1}, g_{i+2}, \ldots, g_{n}\right) \in G \times G^{n-1}, \\
& \boldsymbol{m}_{\{i\}}:=\left(m_{0}, m_{1}, \ldots, m_{i-1}, m_{i}+m_{i+1}, m_{i+2}, \ldots, m_{n}\right) \in M \times M^{n-1} \text { and } \\
& \boldsymbol{m}_{\{\triangleleft i\}}:=\left(m_{0} g_{i+1}, m_{1} g_{i+1}, \ldots, m_{i-1} g_{i+1}, m_{i} g_{i+1}+m_{i+1} \varphi\left(g_{i+1}\right),\right. \\
&\left.m_{i+2} \varphi\left(g_{i+1}\right), \ldots, m_{n} \varphi\left(g_{i+1}\right)\right) \in M \times M^{n-1},
\end{aligned}
$$

where $n$ and $i$ are integers with $n \geq 2$ and $0 \leq i \leq n-1$.

Let $C_{n}^{\mathrm{BR}}(X ; \mathbb{Z})_{X}$ be the free abelian group generated by the elements

$$
(\boldsymbol{g}, \boldsymbol{m})=\left(g_{0}, g_{1}, \ldots, g_{n} ; m_{0}, m_{1}, \ldots, m_{n}\right) \in\left(G \times G^{n}\right) \times\left(M \times M^{n}\right)
$$

if $n \geq 1$, and $C_{n}^{\mathrm{BR}_{\mathrm{U}}}(X ; \mathbb{Z})_{X}:=0$ otherwise.

We define a boundary map $\partial_{n}^{\mathrm{BR}_{\mathrm{U}}}: C_{n}^{\mathrm{BR}_{\mathrm{U}}}(X ; \mathbb{Z})_{X} \rightarrow C_{n-1}^{\mathrm{BR}_{\mathrm{U}}}(X ; \mathbb{Z})_{X}$ by

$$
\partial_{n}^{\mathrm{BR}_{\mathrm{U}}}(\boldsymbol{g} ; \boldsymbol{m})=\sum_{i=0}^{n-1}(-1)^{i}\left\{\left(\boldsymbol{g}_{\{i\}} ; \boldsymbol{m}_{\{i\}}\right)-\left(\boldsymbol{g}_{\{\triangleleft i\}} ; \boldsymbol{m}_{\{\triangleleft i\}}\right)\right\}
$$

if $n \geq 2$, and $\partial_{n}^{\mathrm{BR}}=0$ otherwise. For example, we have

$$
\begin{aligned}
\partial_{3}^{\mathrm{BR}} & \left(g_{0}, g_{1}, g_{2}, g_{3} ; m_{0}, m_{1}, m_{2}, m_{3}\right) \\
= & (-1)^{0}\left\{\left(g_{0}, g_{2}, g_{3} ; m_{0}+m_{1}, m_{2}, m_{3}\right)\right. \\
& \left.\quad-\left(g_{1}^{-1} g_{0} g_{1}, g_{2}, g_{3} ; m_{0} g_{1}+m_{1} \varphi\left(g_{1}\right), m_{2} \varphi\left(g_{1}\right), m_{3} \varphi\left(g_{1}\right)\right)\right\} \\
& +(-1)^{1}\left\{\left(g_{0}, g_{1}, g_{3} ; m_{0}, m_{1}+m_{2}, m_{3}\right)\right. \\
& \left.\quad-\left(g_{2}^{-1} g_{0} g_{2}, g_{2}^{-1} g_{1} g_{2}, g_{3} ; m_{0} g_{2}, m_{1} g_{2}+m_{2} \varphi\left(g_{2}\right), m_{3} \varphi\left(g_{2}\right)\right)\right\} \\
& +(-1)^{2}\left\{\left(g_{0}, g_{1}, g_{2} ; m_{0}, m_{1}, m_{2}+m_{3}\right)\right. \\
& \left.\quad-\left(g_{3}^{-1} g_{0} g_{3}, g_{3}^{-1} g_{1} g_{3}, g_{3}^{-1} g_{2} g_{3} ; m_{0} g_{3}, m_{1} g_{3}, m_{2} g_{3}+m_{3} \varphi\left(g_{3}\right)\right)\right\}
\end{aligned}
$$

Lemma 4.1. $C_{*}^{\mathrm{BR}}(X ; \mathbb{Z})_{X}=\left(C_{n}^{\mathrm{BR}}(X ; \mathbb{Z})_{X}, \partial_{n}^{\mathrm{BR}_{\mathrm{U}}}\right)_{n \in \mathbb{Z}}$ is a chain complex.

4.1.2 The chain map $\gamma: C_{*}^{\mathrm{BR}}(X ; \mathbb{Z})_{X} \rightarrow C_{*}^{\mathrm{BR}}(X ; \mathbb{Z})_{X}$

For $n \geq 1$, define a homomorphism $\gamma_{n}: C_{n}^{\mathrm{BR}}(X ; \mathbb{Z})_{X} \rightarrow C_{n}^{\mathrm{BR}}(X ; \mathbb{Z})_{X}$ by

$$
\gamma_{n}\left(\left(m_{0}, g_{0}\right),\left(m_{1}, g_{1}\right), \ldots,\left(m_{n}, g_{n}\right)\right):=\left(\boldsymbol{g} ; m_{0}^{\prime}, m_{1}^{\prime}, \ldots, m_{n-1}^{\prime}, m_{n}\right),
$$

where we write $m_{i}^{\prime}:=m_{i}-m_{i+1}$ and $\boldsymbol{g}=\left(g_{0}, \ldots, g_{n}\right)$. Define $\gamma_{n}=0$ for $i<1$.

Lemma 4.2. (1) The map $\gamma$ is a chain map, that is, it holds that

$$
\gamma_{n-1} \circ \partial_{n}^{\mathrm{BR}}=\partial_{n}^{\mathrm{BR}} \circ \gamma_{n} .
$$

(2) For each integer $n$, the map $\gamma_{n}$ is an isomorphism. 
4.1.3 The chain map $\psi: C_{*}^{\mathrm{BR}}(X ; \mathbb{Z})_{X} \rightarrow C_{*+1}^{\mathrm{gp}}(M ; \mathbb{Z})_{G}$

For $n \geq 1$, define $\psi_{n}: C_{n}^{\mathrm{BR}}(X ; \mathbb{Z})_{X} \rightarrow C_{n+1}^{\mathrm{gp}}(M ; \mathbb{Z})_{G}$ by

$$
\psi_{n}\left(g_{0}, g_{1}, \ldots, g_{n} ; m_{0}, m_{1}, \ldots, m_{n}\right):=\sum_{\boldsymbol{k} \in \mathcal{K}_{n}}(-1)^{|\boldsymbol{k}|}\left(m_{0} \boldsymbol{g}_{\boldsymbol{k}_{0}}, m_{1} \boldsymbol{g}_{\boldsymbol{k}_{1}}, \ldots, m_{n} \boldsymbol{g}_{\boldsymbol{k}_{n}}\right),
$$

where $\mathcal{K}_{n}:=\{0,1\}^{n}$ and

$$
\begin{aligned}
|\boldsymbol{k}| & :=k_{1}+k_{2}+\cdots+k_{n}, \\
\boldsymbol{g}_{\boldsymbol{k}_{0}} & :=g_{1}^{k_{1}} g_{2}^{k_{2}} \cdots g_{n}^{k_{n}} \in G \text { and } \\
\boldsymbol{g}_{\boldsymbol{k}_{i}} & :=\varphi\left(g_{1}^{k_{1}} g_{2}^{k_{2}} \cdots g_{i}^{k_{i}}\right) g_{i+1}^{k_{i+1}} g_{i+2}^{k_{i+2}} \cdots g_{n}^{k_{n}} \in G
\end{aligned}
$$

for an element $\boldsymbol{k}=\left(k_{1}, \ldots, k_{n}\right) \in \mathcal{K}_{n}$ and an integer $i$ with $1 \leq i \leq n$. Define $\psi_{n}=0$ for $n<1$. We note that the codomain of $\psi_{n}$ is $C_{n+1}^{\mathrm{gp}}(M ; \mathbb{Z})_{G}$.

For example, we have

$$
\begin{aligned}
\psi_{3}(\boldsymbol{g} ; \boldsymbol{m})= & (-1)^{0+1+1}\left(m_{0} g_{1}^{0} g_{2}^{1} g_{3}^{1}, m_{1} \varphi\left(g_{1}^{0}\right) g_{2}^{1} g_{3}^{1}, m_{2} \varphi\left(g_{1}^{0} g_{2}^{1}\right) g_{3}^{1}, m_{3} \varphi\left(g_{1}^{0} g_{2}^{1} g_{3}^{1}\right)\right) \\
& +(-1)^{0+1+0}\left(m_{0} g_{1}^{0} g_{2}^{1} g_{3}^{0}, m_{1} \varphi\left(g_{1}^{0}\right) g_{2}^{1} g_{3}^{0}, m_{2} \varphi\left(g_{1}^{0} g_{2}^{1}\right) g_{3}^{0}, m_{3} \varphi\left(g_{1}^{0} g_{2}^{1} g_{3}^{0}\right)\right) \\
& +(-1)^{0+0+1}\left(m_{0} g_{1}^{0} g_{2}^{0} g_{3}^{1}, m_{1} \varphi\left(g_{1}^{0}\right) g_{2}^{0} g_{3}^{1}, m_{2} \varphi\left(g_{1}^{0} g_{2}^{0}\right) g_{3}^{1}, m_{3} \varphi\left(g_{1}^{0} g_{2}^{0} g_{3}^{1}\right)\right) \\
& +(-1)^{0+0+0}\left(m_{0} g_{1}^{0} g_{2}^{0} g_{3}^{0}, m_{1} \varphi\left(g_{1}^{0}\right) g_{2}^{0} g_{3}^{0}, m_{2} \varphi\left(g_{1}^{0} g_{2}^{0}\right) g_{3}^{0}, m_{3} \varphi\left(g_{1}^{0} g_{2}^{0} g_{3}^{0}\right)\right) \\
& +(-1)^{1+1+1}\left(m_{0} g_{1}^{1} g_{2}^{1} g_{3}^{1}, m_{1} \varphi\left(g_{1}^{1}\right) g_{2}^{1} g_{3}^{1}, m_{2} \varphi\left(g_{1}^{1} g_{2}^{1}\right) g_{3}^{1}, m_{3} \varphi\left(g_{1}^{1} g_{2}^{1} g_{3}^{1}\right)\right) \\
& +(-1)^{1+1+0}\left(m_{0} g_{1}^{1} g_{2}^{1} g_{3}^{0}, m_{1} \varphi\left(g_{1}^{1}\right) g_{2}^{1} g_{3}^{0}, m_{2} \varphi\left(g_{1}^{1} g_{2}^{1}\right) g_{3}^{0}, m_{3} \varphi\left(g_{1}^{1} g_{2}^{1} g_{3}^{0}\right)\right) \\
& +(-1)^{1+0+1}\left(m_{0} g_{1}^{1} g_{2}^{0} g_{3}^{1}, m_{1} \varphi\left(g_{1}^{1}\right) g_{2}^{0} g_{3}^{1}, m_{2} \varphi\left(g_{1}^{1} g_{2}^{0}\right) g_{3}^{1}, m_{3} \varphi\left(g_{1}^{1} g_{2}^{0} g_{3}^{1}\right)\right) \\
& +(-1)^{1+0+0}\left(m_{0} g_{1}^{1} g_{2}^{0} g_{3}^{0}, m_{1} \varphi\left(g_{1}^{1}\right) g_{2}^{0} g_{3}^{0}, m_{2} \varphi\left(g_{1}^{1} g_{2}^{0}\right) g_{3}^{0}, m_{3} \varphi\left(g_{1}^{1} g_{2}^{0} g_{3}^{0}\right)\right) .
\end{aligned}
$$

Lemma 4.3. The map $\psi=\left(\psi_{n}\right)$ is a chain map, that is, it holds that

$$
\psi_{n-1} \circ \partial_{n}^{\mathrm{BR}}=\partial_{n+1}^{\mathrm{gp}} \circ \psi_{n} .
$$

\subsection{Cocycles of $G$-Alexander biquandles with the $X$-set $X$}

As a consequence of Subsection 4.1 we have a sequence

$$
C_{n}^{\mathrm{BR}}(X ; \mathbb{Z})_{X} \stackrel{\gamma_{n}}{\longrightarrow} C_{n}^{\mathrm{BR}_{\mathrm{U}}}(X ; \mathbb{Z})_{X} \stackrel{\psi_{n}}{\longrightarrow} C_{n+1}^{\mathrm{gp}}(M ; \mathbb{Z})_{G}
$$

of chain groups $C_{n}^{\mathrm{BR}}(X ; \mathbb{Z})_{X}, C_{n}^{\mathrm{BR}}(X ; \mathbb{Z})_{X}, C_{n}^{\mathrm{gp}}(M ; \mathbb{Z})_{G}$ and chain maps $\gamma, \psi$ for $n \geq 1$. Therefore we have the following theorem.

Theorem 4.4. For any $(n+1)$-cocycle $f: C_{n+1}^{\mathrm{gp}}(M ; \mathbb{Z})_{G} \rightarrow A$, the map

$$
\Phi_{f}:=f \circ \psi_{n} \circ \gamma_{n}: C_{n}^{\mathrm{BR}}(X ; \mathbb{Z})_{X} \rightarrow A
$$

is a birack $n$-cocycle of the $G$-Alexander biquandle $X=M \times G$.

Theorem 4.5. (1) Let $f: M^{3} \rightarrow A$ be a $G$-invariant $A$-multilinear map. The birack 2 -cocycle $\Phi_{f}=f \circ \psi_{2} \circ \gamma_{2}: C_{2}^{\mathrm{BR}}(X ; \mathbb{Z})_{X} \rightarrow A$ of the $G$-Alexander biquandle $X=M \times G$ is formulated as

$$
\begin{aligned}
& \Phi_{f}\left(\left(m_{0}, g_{0}\right),\left(m_{1}, g_{1}\right),\left(m_{2}, g_{2}\right)\right) \\
& =f\left(m_{0}^{\prime}\left(1-\varphi\left(g_{1}\right)^{-1} g_{1}\right), m_{1}^{\prime}, m_{2}\left(1-\varphi\left(g_{2}\right) g_{2}^{-1}\right)\right)
\end{aligned}
$$

for $\left(\left(m_{0}, g_{0}\right),\left(m_{1}, g_{1}\right),\left(m_{2}, g_{2}\right)\right) \in X \times X^{2} \subset C_{2}^{\mathrm{BR}}(X ; \mathbb{Z})_{X}$, where $m_{i}^{\prime}:=$ $m_{i}-m_{i+1}$. 
(2) Let $f: M^{4} \rightarrow A$ be a G-invariant A-multilinear map. The birack 3cocycle $\Phi_{f}=f \circ \psi_{3} \circ \gamma_{3}: C_{3}^{\mathrm{BR}}(X ; \mathbb{Z})_{X} \rightarrow A$ of the $G$-Alexander biquandle $X=M \times G$ is formulated as

$$
\begin{aligned}
& \Phi_{f}\left(\left(m_{0}, g_{0}\right),\left(m_{1}, g_{1}\right),\left(m_{2}, g_{2}\right),\left(m_{3}, g_{3}\right)\right) \\
& =f\left(m_{0}^{\prime}\left(1-\varphi\left(g_{1}\right)^{-1} g_{1}\right), m_{1}^{\prime}, m_{2}^{\prime}, m_{3}\left(1-\varphi\left(g_{3}\right) g_{3}^{-1}\right)\right) \\
& \quad-f\left(m_{0}^{\prime}\left(1-\varphi\left(g_{1}\right)^{-1} g_{1}\right) g_{2}, m_{1}^{\prime} g_{2}, m_{2}^{\prime} \varphi\left(g_{2}\right), m_{3}\left(1-\varphi\left(g_{3}\right) g_{3}^{-1}\right) \varphi\left(g_{2}\right)\right)
\end{aligned}
$$

for $\left(\left(m_{0}, g_{0}\right),\left(m_{1}, g_{1}\right),\left(m_{2}, g_{2}\right),\left(m_{3}, g_{3}\right)\right) \in X \times X^{3} \subset C_{3}^{\mathrm{BR}}(X ; \mathbb{Z})_{X}$, where $m_{i}^{\prime}:=m_{i}-m_{i+1}$.

\section{Cocycles of $G$-Alexander multiple conjugation biquandles}

Throughout this section, let $X=\bigsqcup_{m \in M}(\{m\} \times G)=M \times G$ be the $G$-Alexander multiple conjugation biquandle of $(M, \varphi)$, see Definition 2.5. Our goal in this section is to give Theorem 5.7

\subsection{Degenerate subcomplexes $D_{*}^{\mathrm{BR}}(X ; \mathbb{Z}), D_{*}^{\mathrm{BR}_{\mathrm{U}}}(X ; \mathbb{Z})$ and the induced homomorphisms $\gamma_{n}, \psi_{n, \lambda}$}

5.1.1 The degenerate subcomplex $D_{*}^{\mathrm{BR}}(X ; \mathbb{Z})$ of $C_{*}^{\mathrm{BR}}(X ; \mathbb{Z})$

Let $D_{n}^{\mathrm{BR}}(X ; \mathbb{Z})$ be the subgroup of $C_{n}^{\mathrm{BR}}(X ; \mathbb{Z})$ generated by the elements of the following two sets

$$
\begin{aligned}
& \bigcup_{i=1}^{n-1}\left\{\left(\boldsymbol{x}^{i-1},(m, g),(m, h), \boldsymbol{x}_{i+2}\right) \mid \boldsymbol{x} \in X^{n}, m \in M, g, h \in G\right\} \text { and } \\
& \bigcup_{i=1}^{n}\left\{\begin{array}{ll}
\left(\boldsymbol{x}^{i-1},(m, g h), \boldsymbol{x}_{i+1}\right)-\left(\boldsymbol{x}^{i-1},(m, g), \boldsymbol{x}_{i+1}\right) & \boldsymbol{x} \in X^{n}, \\
-\left(\boldsymbol{x}^{i-1} \underset{*}{ }(m, g),\left((m, h), \boldsymbol{x}_{i+1}\right) \bar{*}(m, g)\right) & m \in M, g, h \in G
\end{array}\right\}
\end{aligned}
$$

for $n \geq 2$. We define $D_{n}^{\mathrm{BR}}(X ; \mathbb{Z})=0$ for $n \leq 1$. We note that

$$
\begin{aligned}
& \left(\boldsymbol{x}^{i-1} *(m, g),\left((m, h), \boldsymbol{x}_{i+1}\right) \approx(m, g)\right) \\
& =\left(x_{1} \underline{*}(m, g), \ldots, x_{i-1} \underline{*}(m, g),(m, h) \approx(m, g), x_{i+1} \text { * }(m, g), \ldots, x_{n} \bar{*}(m, g)\right) .
\end{aligned}
$$

Lemma 5.1. $D_{*}^{\mathrm{BR}}(X ; \mathbb{Z}):=\left(D_{n}^{\mathrm{BR}}(X ; \mathbb{Z}), \partial_{n}^{\mathrm{BR}}\right)_{n \in \mathbb{Z}}$ is a subcomplex of $C_{*}^{\mathrm{BR}}(X ; \mathbb{Z})$.

Proof. We fix an integer $n \geq 2$ and show $\partial_{n}^{\mathrm{BR}}\left(D_{n}^{\mathrm{BR}}(X ; \mathbb{Z})\right) \subset D_{n-1}^{\mathrm{BR}}(X ; \mathbb{Z})$. It suffices to show

$$
\partial_{n}^{\mathrm{BR}}\left(\boldsymbol{x}^{i-1},(m, g),(m, h), \boldsymbol{x}_{i+2}\right) \equiv 0
$$

for any $i$ with $1 \leq i \leq n-1$ and

$$
\begin{aligned}
\partial_{n}^{\mathrm{BR}}\left(\boldsymbol{x}^{i-1},(m, g h), \boldsymbol{x}_{i+1}\right) \equiv & \partial_{n}^{\mathrm{BR}}\left(\boldsymbol{x}^{i-1},(m, g), \boldsymbol{x}_{i+1}\right) \\
& +\partial_{n}^{\mathrm{BR}}\left(\boldsymbol{x}^{i-1} \underline{*}(m, g),\left((m, h), \boldsymbol{x}_{i+1}\right) \star(m, g)\right)
\end{aligned}
$$

for any $i$ with $1 \leq i \leq n$ in $C_{n-1}^{\mathrm{BR}}(X ; \mathbb{Z}) / D_{n-1}^{\mathrm{BR}}(X ; \mathbb{Z})$. 
We verify the first equality in the quotient group. Put $x_{i}:=(m, g), x_{i+1}:=$ $(m, h)$ and $\boldsymbol{x}:=\left(x_{1}, \ldots, x_{n}\right)$.

$$
\begin{aligned}
& \partial_{n}^{\mathrm{BR}}(\boldsymbol{x}) \\
& =\sum_{j=1}^{n}(-1)^{j-1}\left\{\left(\boldsymbol{x}^{j-1}, \boldsymbol{x}_{j+1}\right)-\left(\boldsymbol{x}^{j-1} \underset{*}{x_{j}, \boldsymbol{x}_{j+1}} \bar{\aleph}_{j}\right)\right\} \\
& =\sum_{j=1}^{i-1}(-1)^{j-1} \underline{\left\{\left(\boldsymbol{x}^{j-1}, \boldsymbol{x}_{j+1}\right)-\left(\boldsymbol{x}^{j-1} \underline{*} x_{j}, \boldsymbol{x}_{j+1} \approx x_{j}\right)\right\}}(A) \\
& +(-1)^{i-1} \underline{\left\{\left(\boldsymbol{x}^{i-1},(m, h), \boldsymbol{x}_{i+2}\right)-\left(\boldsymbol{x}^{i-1} \underline{*}(m, g),\left((m, h), \boldsymbol{x}_{i+2}\right) \mp(m, g)\right)\right\}}(B) \\
& +(-1)^{i} \underline{\left\{\left(\boldsymbol{x}^{i-1},(m, g), \boldsymbol{x}_{i+2}\right)-\left(\left(\boldsymbol{x}^{i-1},(m, g)\right) \underline{*}(m, h), \boldsymbol{x}_{i+2} \bar{*}(m, h)\right)\right\}}(C) \\
& +\sum_{j=i+2}^{n}(-1)^{j-1} \underline{\left\{\left(\boldsymbol{x}^{j-1}, \boldsymbol{x}_{j+1}\right)-\left(\boldsymbol{x}^{j-1} \underline{*} x_{j}, \boldsymbol{x}_{j+1} \bar{*} x_{j}\right)\right\}}(D) .
\end{aligned}
$$

Since $(m, g h)=\left(m, h\left(h^{-1} g h\right)\right)$ and $(m, g) \underline{*}(m, h)=(m h+m(\varphi(h)-$ $\left.h), h^{-1} g h\right)=\left(m, h^{-1} g h\right) \mp(m, h)$, we see that for $(B)$ and $(C)$ of (19),

$$
\begin{aligned}
& (B)-(C) \\
& =\left(\boldsymbol{x}^{i-1},(m, h), \boldsymbol{x}_{i+2}\right)-\left(\boldsymbol{x}^{i-1} \text { * }(m, g),\left((m, h), \boldsymbol{x}_{i+2}\right) \bar{*}(m, g)\right)-\left(\boldsymbol{x}^{i-1},(m, g), \boldsymbol{x}_{i+2}\right) \\
& \quad+\left(\left(\boldsymbol{x}^{i-1},(m, g)\right) \text { }(m, h), \boldsymbol{x}_{i+2} \bar{*}(m, h)\right) \\
& \equiv\left(\boldsymbol{x}^{i-1},(m, h), \boldsymbol{x}_{i+2}\right)-\left(\boldsymbol{x}^{i-1},(m, g h), \boldsymbol{x}_{i+2}\right) \\
& \quad+\left(\left(\boldsymbol{x}^{i-1},(m, g)\right) \text { * }(m, h), \boldsymbol{x}_{i+2} \bar{*}(m, h)\right) \\
& =\left(\boldsymbol{x}^{i-1},(m, h), \boldsymbol{x}_{i+2}\right)-\left(\boldsymbol{x}^{i-1},\left(m, h\left(h^{-1} g h\right)\right), \boldsymbol{x}_{i+2}\right) \\
& \quad+\left(\boldsymbol{x}^{i-1} \underline{*}(m, h),\left(\left(m, h^{-1} g h\right), \boldsymbol{x}_{i+2}\right) \bar{*}(m, h)\right) \\
& \equiv 0 .
\end{aligned}
$$

When $j \neq i$ and $j \neq i+1$, we have $\left(\boldsymbol{x}_{j-1}, \boldsymbol{x}^{j+1}\right) \equiv 0$ immediately. Since the first element of $(m, g) \leqslant x_{j}$ is equal to that of $(m, h) \underline{*} x_{j}$ and the first element of $(m, g) \mp x_{j}$ is equal to that of $(m, h) \mp x_{j}$, we have

$$
\begin{aligned}
& \left(\boldsymbol{x}^{j-1} x_{j},\left(\boldsymbol{x}_{j+1}^{i-1},(m, g),(m, h), \boldsymbol{x}^{i+2}\right) \bar{*} x_{j}\right) \in D_{n-1}^{\mathrm{BR}}(X ; \mathbb{Z}) \text { and } \\
& \left(\left(\boldsymbol{x}^{i-1},(m, g),(m, h), \boldsymbol{x}_{i+2}^{j-1}\right) \underline{*} x_{j}, \boldsymbol{x}^{j+1} \bar{*} x_{j}\right) \in D_{n-1}^{\mathrm{BR}}(X ; \mathbb{Z}),
\end{aligned}
$$

where $\boldsymbol{x}_{a}^{b}$ means the sequence $x_{a}, x_{a+1}, \ldots, x_{b-1}, x_{b}$. Then, $\left(\boldsymbol{x}_{j-1} \underline{*} x_{j}, \boldsymbol{x}^{j+1} \bar{*}\right.$ $\left.x_{i}\right) \equiv 0$ in the equality of (19). Then, we have $(A) \equiv(D) \equiv 0$. Hence, we have $\partial_{n}^{\mathrm{BR}}(\boldsymbol{x}) \equiv 0$.

We verify the second equality in the quotient group. Put $x_{i}:=(m, g h)$ and 
$\boldsymbol{x}:=\left(x_{1}, \ldots, x_{n}\right)=\left(x_{1}, \ldots, x_{i-1},(m, g h), x_{i+1}, \ldots, x_{n}\right)$. We have

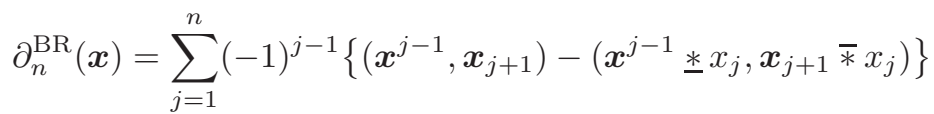

$$
\begin{aligned}
& =\sum_{j=1}^{i-1}(-1)^{j-1}\left\{\left(\boldsymbol{x}^{j-1}, \boldsymbol{x}_{j+1}\right)-\left(\boldsymbol{x}^{j-1} \underline{*} x_{j}, \boldsymbol{x}_{j+1} \bar{*} x_{j}\right)\right\} \\
& +\sum_{j=i}^{i}(-1)^{j-1}\left\{\left(\boldsymbol{x}^{j-1}, \boldsymbol{x}_{j+1}\right)-\left(\boldsymbol{x}^{j-1} \underset{*}{x_{j}}, \boldsymbol{x}_{j+1} \bar{*} x_{j}\right)\right\} \\
& +\sum_{j=i+1}^{n}(-1)^{j-1}\left\{\left(\boldsymbol{x}^{j-1}, \boldsymbol{x}_{j+1}\right)-\left(\boldsymbol{x}^{j-1} \underline{*} x_{j}, \boldsymbol{x}_{j+1} \bar{*} x_{j}\right)\right\} .
\end{aligned}
$$

When $1 \leq j \leq i-1$, we have

$$
\begin{aligned}
\left(\boldsymbol{x}^{j-1}, \boldsymbol{x}_{j+1}\right)= & \left(\boldsymbol{x}^{j-1}, \boldsymbol{x}_{j+1}^{i-1},(m, g h), \boldsymbol{x}_{i+1}\right) \\
\equiv & \underline{\left(\boldsymbol{x}^{j-1}, \boldsymbol{x}_{j+1}^{i-1},(m, g), \boldsymbol{x}_{i+1}\right)}(A) \\
& +\underline{\left(\left(\boldsymbol{x}^{j-1}, \boldsymbol{x}_{j+1}^{i-1}\right) \underline{*}(m, g),\left((m, h), \boldsymbol{x}_{i+1}\right) Æ(m, g)\right)}(B)
\end{aligned}
$$

Put $x_{j}:=\left(m_{j}, g_{j}\right)$. Since $\left(m, g^{\prime}\right) \mp x_{j}=\left(m \varphi\left(g_{j}\right), g^{\prime}\right)$ for any $g^{\prime} \in G$, we have

$$
\begin{aligned}
& \left(\boldsymbol{x}^{j-1} \underline{*} x_{j}, \boldsymbol{x}_{j+1} \mp x_{j}\right) \\
& =\left(\boldsymbol{x}^{j-1} \underline{*} x_{j},\left(\boldsymbol{x}_{j+1}^{i-1},(m, g h), \boldsymbol{x}_{i+1}\right) \mp x_{j}\right) \\
& =\left(\boldsymbol{x}^{j-1} \underline{*} x_{j}, \boldsymbol{x}_{j+1}^{i-1} \bar{*} x_{j},\left(m \varphi\left(g_{j}\right), g h\right), \boldsymbol{x}_{i+1} \bar{*} x_{j}\right) \\
& \equiv\left(\boldsymbol{x}^{j-1} \underline{*} x_{j}, \boldsymbol{x}_{j+1}^{i-1} \mp x_{j},\left(m \varphi\left(g_{j}\right), g\right), \boldsymbol{x}_{i+1} \bar{*} x_{j}\right) \\
& +\left(\left(\boldsymbol{x}^{j-1} \underline{*} x_{j}, \boldsymbol{x}_{j+1}^{i-1} \bar{*} x_{j}\right) \underline{*}\left(m \varphi\left(g_{j}\right), g\right),\left(\left(m \varphi\left(g_{j}\right), h\right), \boldsymbol{x}_{i+1} \bar{*} x_{j}\right) \text { ж }\left(m \varphi\left(g_{j}\right), g\right)\right) \\
& =\underline{\left(\boldsymbol{x}^{j-1} \underline{*} x_{j},\left(\boldsymbol{x}_{j+1}^{i-1},(m, g), \boldsymbol{x}_{i+1}\right) \approx x_{j}\right)}(C)
\end{aligned}
$$

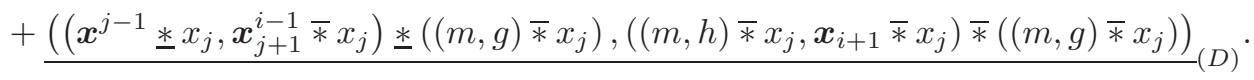

Then, we have

$$
(D)=\underline{\left(\left(\boldsymbol{x}^{j-1} \underline{*}(m, g)\right) \underline{*}\left(x_{j} \underline{*}(m, g)\right),\left(\boldsymbol{x}_{j+1}^{i-1} \underline{*}(m, g),(m, h) \bar{*}(m, g), \boldsymbol{x}_{i+1} \bar{*}(m, g)\right) \bar{*}\left(x_{j} \underline{*}(m, g)\right)\right)}\left(D^{\prime}\right) .
$$

When $j=i$, we have

$$
\begin{aligned}
& \left(\boldsymbol{x}^{j-1}, \boldsymbol{x}_{j+1}\right)=\underline{\left(\boldsymbol{x}^{i-1}, \boldsymbol{x}_{i+1}\right)}(E), \\
& \left(\boldsymbol{x}^{j-1} \underline{*} x_{j}, \boldsymbol{x}_{j+1} \bar{*} x_{j}\right) \\
& =\left(\boldsymbol{x}^{i-1} \Vdash(m, g h), \boldsymbol{x}_{i+1} \bar{*}(m, g h)\right) \\
& ={\underline{\left(\left(\boldsymbol{x}^{i-1} \underline{*}(m, g)\right) \underline{*}((m, h) \bar{*}(m, g)),\left(\boldsymbol{x}_{i+1} \bar{*}(m, g)\right) \mp((m, h) \mp(m, g))\right)}}_{(F)} .
\end{aligned}
$$

When $i+1 \leq j \leq n$, we have 


$$
\begin{aligned}
\left(\boldsymbol{x}^{j-1}, \boldsymbol{x}_{j+1}\right)= & \left(\boldsymbol{x}^{i-1},(m, g h), \boldsymbol{x}_{i+1}^{j-1}, \boldsymbol{x}_{j+1}\right) \\
\equiv & \underline{\left(\boldsymbol{x}^{i-1},(m, g), \boldsymbol{x}_{i+1}^{j-1}, \boldsymbol{x}_{j+1}\right)}(G) \\
& +\underline{\left(\boldsymbol{x}^{i-1} \underline{*}(m, g),\left\{(m, h), \boldsymbol{x}_{i+1}^{j-1}, \boldsymbol{x}_{j+1}\right\} Æ(m, g)\right)}(H)
\end{aligned}
$$

Put $x_{j}:=\left(m_{j}, g_{j}\right)$. We have $\left(m^{\prime}, g^{\prime}\right) \underline{*} x_{j}=\left(m^{\prime} g_{j}+m_{j}\left(\varphi\left(g_{j}\right)-g_{j}\right), g_{j}^{-1} g^{\prime} g_{j}\right)$ and $\left(m^{\prime}, g^{\prime}\right) \approx m_{j}=\left(m^{\prime} \varphi\left(g_{j}\right), g^{\prime}\right)$ for any $\left(m^{\prime}, g^{\prime}\right) \in X$. Put $A:=m g_{j}+m_{j}\left(\varphi\left(g_{j}\right)-g_{j}\right)$. We have

$$
\begin{aligned}
& \left(\boldsymbol{x}^{j-1} \underline{*} x_{j}, \boldsymbol{x}_{j+1} \bar{*} x_{j}\right) \\
& =\left(\boldsymbol{x}^{i-1} \underline{*} x_{j},\left(A, g_{j}^{-1} g g_{j} g_{j}^{-1} h g_{j}\right), \boldsymbol{x}_{i+1}^{j-1} \Perp x_{j}, \boldsymbol{x}_{j+1} \mp x_{j}\right) \\
& \equiv\left(\boldsymbol{x}^{i-1} \underline{*} x_{j},\left(A, g_{j}^{-1} g g_{j}\right), \boldsymbol{x}_{i+1}^{j-1} \Perp x_{j}, \boldsymbol{x}_{j+1} \bar{*} x_{j}\right) \\
& +\left(\left(\boldsymbol{x}^{i-1} \underline{*} x_{j}\right) \underline{*}\left(A, g_{j}^{-1} g g_{j}\right),\left(\left(A, g_{j}^{-1} h g_{j}\right), \boldsymbol{x}_{i+1}^{j-1} \underline{*} x_{j}, \boldsymbol{x}_{j+1} \bar{*} x_{j}\right) \mp\left(A, g_{j}^{-1} g g_{j}\right)\right)
\end{aligned}
$$

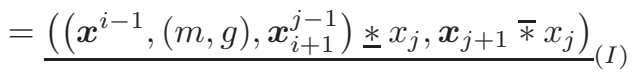

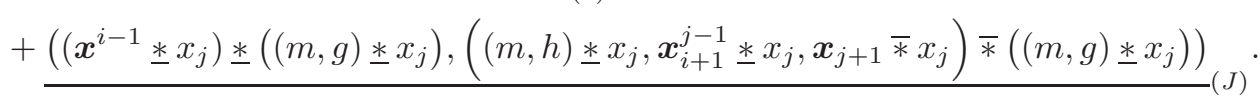

Then, we have

$J=$

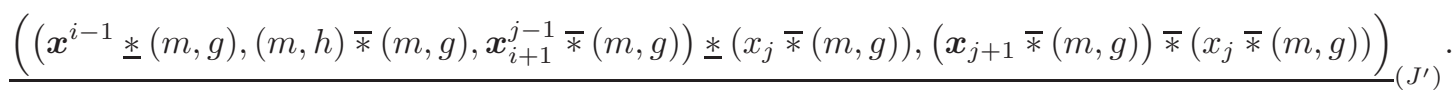

Hence, we have

$$
\begin{aligned}
\partial_{n}^{\mathrm{BR}}(\boldsymbol{x}) & \equiv \sum_{j=1}^{i-1}(-1)^{j-1}\left(A+B-\left(C+D^{\prime}\right)\right) \\
& +(-1)^{i-1}(E-F)+\sum_{j=i+1}^{n}(-1)^{j-1}\left(G+H-\left(I+J^{\prime}\right)\right) .
\end{aligned}
$$

By the definition of the map $\partial_{n}^{\text {BR }}$, we have

$$
\begin{aligned}
\partial_{n}^{\mathrm{BR}}\left(\boldsymbol{x}^{i-1},(m, g), \boldsymbol{x}_{i+1}\right)=\sum_{j=1}^{i-1}(-1)^{j-1}(A-C) \\
\quad+(-1)^{i-1}\left(E-\left(\boldsymbol{x}^{i-1} \underline{*}(m, g), \boldsymbol{x}_{i+1} \bar{*}(m, g)\right)\right)+\sum_{j=i+1}^{n}(-1)^{j-1}(G-I)
\end{aligned}
$$

and

$$
\begin{aligned}
\partial_{n}^{\mathrm{BR}}\left(\boldsymbol{x}^{i-1} \underset{*}{(m, g)},\left((m, h), \boldsymbol{x}_{i+1}\right) \bar{*}(m, g)\right)=\sum_{j=1}^{i-1}(-1)^{j-1}\left(B-D^{\prime}\right) \\
\quad+(-1)^{i-1}\left(\left(\boldsymbol{x}^{i-1} \underset{*}{(m, g), \boldsymbol{x}_{i+1}} \bar{*}(m, g)\right)-F\right)+\sum_{j=i+1}^{n}(-1)^{j-1}\left(H-J^{\prime}\right) .
\end{aligned}
$$


Therefore, it holds that

$$
\partial_{n}^{\mathrm{BR}}(\boldsymbol{x}) \equiv \partial_{n}^{\mathrm{BR}}\left(\boldsymbol{x}^{i-1},(m, g), \boldsymbol{x}_{i+1}\right)+\partial_{n}^{\mathrm{BR}}\left(\boldsymbol{x}^{i-1} \underline{*}(m, g),\left((m, h), \boldsymbol{x}_{i+1}\right) \bar{*}(m, g)\right) .
$$

This completes the proof.

The normalized birack chain complex is $C_{*}^{\text {norBR }}(X ; \mathbb{Z}):=C_{*}^{\mathrm{BR}}(X ; \mathbb{Z}) / D_{*}^{\mathrm{BR}}(X ; \mathbb{Z})$. It determines the normalized birack homology group $H_{n}^{\text {norBR }}(X ; \mathbb{Z})$. In the ordinary way, for an abelian group $A$, we have the (co)homology theory with the coefficient group $A$ and the homology group $H_{n}^{\text {norBR }}(X ; A)$ and the cohomology group $H_{\text {norBR }}^{n}(X ; A)$ are defined.

\subsubsection{The degenerate subcomplex $D_{*}^{\mathrm{BR}}(X ; \mathbb{Z})$ of $C_{*}^{\mathrm{BR}}(X ; \mathbb{Z})$}

We introduce the degenerate subcomplex $D_{*}^{\mathrm{BR}}(X ; \mathbb{Z})$ of $C_{*}^{\mathrm{BR}}(X ; \mathbb{Z})$, which is a counterpart of the degenerate subcomplex $D_{*}^{\mathrm{BR}}(X ; \mathbb{Z})$ of $C_{*}^{\mathrm{BR}}(X ; \mathbb{Z})$.

Let $D_{n}^{\mathrm{BR}}(X ; \mathbb{Z})$ be the subgroup of $C_{n}^{\mathrm{BR}_{\mathrm{U}}}(X ; \mathbb{Z})$ generated by the elements of the following sets

$$
\begin{aligned}
& \bigcup_{i=1}^{n-1}\left\{\begin{array}{l|l}
\left.\left(\boldsymbol{g} ; \boldsymbol{m}^{i-1}, 0, \boldsymbol{m}_{i+1}\right) \mid \boldsymbol{g} \in G^{n}, \boldsymbol{m} \in M^{n}\right\} \text { and } \\
\bigcup_{i=1}^{n}\left\{\begin{array}{ll}
\left(\boldsymbol{g}^{i-1}, g_{i} h, \boldsymbol{g}_{i+1} ; \boldsymbol{m}\right) & h \in G, \boldsymbol{g} \in G^{n}, \\
-(\boldsymbol{g} ; \boldsymbol{m})-\left(g_{i}^{-1} \boldsymbol{g}^{i-1} g_{i}, h, \boldsymbol{g}_{i+1} ; \boldsymbol{m}^{i-1} g_{i}, \boldsymbol{m}_{i} \varphi\left(g_{i}\right)\right) & \boldsymbol{m} \in M^{n}
\end{array}\right\}
\end{array}\right.
\end{aligned}
$$

for $n \geq 2$, where we write

$$
\begin{aligned}
&\left(\boldsymbol{g} ; \boldsymbol{m}^{i-1}, 0, \boldsymbol{m}_{i+1}\right):=\left(g_{1}, \ldots, g_{n} ; m_{1}, \ldots, m_{i-1}, 0, m_{i+1}, \ldots, m_{n}\right), \\
&\left(\boldsymbol{g}^{i-1}, g_{i} h, \boldsymbol{g}_{i+1} ; \boldsymbol{m}\right):=\left(g_{1}, \ldots, g_{i-1}, g_{i} h, g_{i+1}, \ldots, g_{n} ; m_{1}, \ldots, m_{n}\right) \text { and } \\
&\left(g_{i}^{-1} \boldsymbol{g}^{i-1} g_{i}, h, \boldsymbol{g}_{i+1} ; \boldsymbol{m}^{i-1} g_{i}, \boldsymbol{m}_{i} \varphi\left(g_{i}\right)\right):=\left(\left(g_{i}^{-1} g_{1} g_{i}\right), \ldots,\left(g_{i}^{-1} g_{i-1} g_{i}\right),\right. \\
&\left.\quad h, g_{i+1}, \ldots, g_{n} ; m_{1} g_{i}, \ldots, m_{i-1} g_{i}, m_{i} \varphi\left(g_{i}\right), \ldots, m_{n} \varphi\left(g_{i}\right)\right) .
\end{aligned}
$$

We define $D_{n}^{\mathrm{BR}}(X ; \mathbb{Z})=0$ for $n \leq 1$.

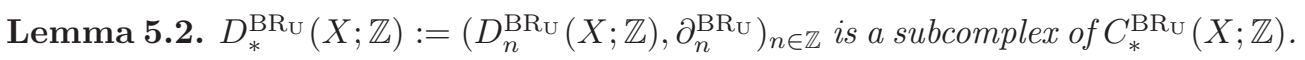

Proof. In Lemma 5.3, we will show that the isomorphism $\gamma_{n}$ defined in Subsection 3.1 gives an isomorphism $D_{n}^{\mathrm{BR}}(X ; \mathbb{Z}) \cong D_{n}^{\mathrm{BR}_{\mathrm{U}}}(X ; \mathbb{Z})$.

The normalized U-birack chain complex is $C_{*}^{\mathrm{norBR}_{\mathrm{U}}}(X ; \mathbb{Z}):=C_{*}^{\mathrm{BR}}(X ; \mathbb{Z}) / D_{*}^{\mathrm{BR}}(X ; \mathbb{Z})$. It determines the homology group $H_{n}^{\text {norBR }}(X ; \mathbb{Z})$. In the ordinary way, for an abelian group $A, H_{n}^{\text {norBR }}(X ; A)$ and $H_{\text {norBR }_{\mathrm{U}}}(X ; A)$ are defined.

\subsubsection{The induced homomorphism $\gamma_{n}$}

Next lemma shows that the isomorphism $\gamma_{n}: C_{n}^{\mathrm{BR}}(X ; \mathbb{Z}) \rightarrow C_{n}^{\mathrm{BR}_{\mathrm{U}}}(X ; \mathbb{Z})$, which is defined in Subsection 3.1 induces the isomorphism $\gamma_{n}: C_{n}^{\text {norBR }}(X ; \mathbb{Z}) \rightarrow$ $C_{n}^{\text {norBR }}(X ; \mathbb{Z})$, where we denote it by the same symbol $\gamma_{n}$ for simplicity.

Lemma 5.3. It holds that $\gamma_{n}\left(D_{n}^{\mathrm{BR}}(X ; \mathbb{Z})\right)=D_{n}^{\mathrm{BR}_{\mathrm{U}}}(X ; \mathbb{Z})$. Therefore, $\gamma_{n}$ induces the isomorphism

$$
\gamma_{n}: C_{n}^{\text {norBR }}(X ; \mathbb{Z}) \rightarrow C_{n}^{\text {norBR }_{\mathrm{U}}}(X ; \mathbb{Z}) .
$$


Proof. Let $\boldsymbol{x} \in D_{n}^{\mathrm{BR}}(X ; \mathbb{Z})$. If $\boldsymbol{x}=\left(\boldsymbol{x}^{i-1},\left(m, g_{i}\right),\left(m, g_{i+1}\right), \boldsymbol{x}_{i+2}\right)$, then we put $\gamma_{n}(\boldsymbol{x})=\left(g_{1}, \ldots, g_{n} ; m_{1} \ldots, m_{n}\right)$. By the definition of $\gamma_{n}$, we have $m_{i}=m-m=$

0 . Then, $\gamma_{n}(\boldsymbol{x}) \in D_{n}^{\mathrm{BR}}(X ; \mathbb{Z})$.

Suppose that

$$
\begin{aligned}
\boldsymbol{x}= & \left(\boldsymbol{x}^{i-1},\left(m_{i}, g_{i} h\right), \boldsymbol{x}_{i+1}\right)-\left(\boldsymbol{x}^{i-1},\left(m_{i}, g_{i}\right), \boldsymbol{x}_{i+1}\right) \\
& -\left(\boldsymbol{x}^{i-1} \underline{*}\left(m_{i}, g_{i}\right),\left(\left(m_{i}, h\right), \boldsymbol{x}_{i+1}\right) \mp\left(m_{i}, g_{i}\right)\right) .
\end{aligned}
$$

We have

$$
\begin{array}{r}
\left(\boldsymbol{x}^{i-1} \underset{*}{ }\left(m_{i}, g_{i}\right),\left(\left(m_{i}, h\right), \boldsymbol{x}_{i+1}\right) \mp\left(m_{i}, g_{i}\right)\right)=\left(\left(A_{1}, g_{i}^{-1} g_{1} g_{i}\right), \ldots,\left(A_{i-1}, g_{i}^{-1} g_{i-1} g_{i}\right),\right. \\
\left.\left(m_{i} \varphi\left(g_{i}\right), h\right),\left(m_{i+1} \varphi\left(g_{i}\right), g_{i+1}\right), \ldots,\left(m_{n} \varphi\left(g_{i}\right), g_{n}\right)\right),
\end{array}
$$

where $A_{j}=m_{j} g_{i}+m_{i}\left(\varphi\left(g_{i}\right)-g_{i}\right)$. We note that $A_{j}-A_{j+1}=\left(m_{j}-m_{j+1}\right) g_{i}$ for $j$ with $1 \leq j<i-1$ and $A_{i-1}-m_{i} \varphi\left(g_{i}\right)=\left(m_{i-1}-m_{i}\right) g_{i}$. Then,

$$
\begin{aligned}
& \gamma_{n}\left(\left(\boldsymbol{x}^{i-1} \underline{*}\left(m_{i}, g_{i}\right),\left(\left(m_{i}, h\right), \boldsymbol{x}_{i+1}\right) \mp\left(m_{i}, g_{i}\right)\right)\right) \\
& =\left(g_{i}^{-1} \boldsymbol{g}^{i-1} g_{i}, h, \boldsymbol{g}_{i+1} ; m_{1}^{\prime} g_{i}, \ldots, m_{i-1}^{\prime} g_{i}, m_{i}^{\prime} \varphi\left(g_{i}\right), \ldots, m_{n-1}^{\prime} \varphi\left(g_{i}\right), m_{n} \varphi\left(g_{i}\right)\right),
\end{aligned}
$$

where $m_{i}^{\prime}:=m_{i}-m_{i+1}$. Then, we have

$$
\begin{aligned}
\gamma_{n}(\boldsymbol{x})= & \left(\boldsymbol{g}^{i-1}, g_{i} h, \boldsymbol{g}_{i+1} ; m_{1}^{\prime}, \ldots, m_{n-1}^{\prime}, m_{n}\right)-\left(\boldsymbol{g} ; m_{1}^{\prime}, \ldots, m_{n-1}^{\prime}, m_{n}\right) \\
& -\left(g_{i}^{-1} \boldsymbol{g}^{i-1} g_{i}, h, \boldsymbol{g}_{i+1} ; m_{1}^{\prime} g_{i}, \ldots, m_{i-1}^{\prime} g_{i},\right. \\
& \left.m_{i}^{\prime} \varphi\left(g_{i}\right), \ldots, m_{n-1}^{\prime} \varphi\left(g_{i}\right), m_{n} \varphi\left(g_{i}\right)\right) \in D_{n}^{\mathrm{BR}}(X ; \mathbb{Z}) .
\end{aligned}
$$

Hence $\gamma_{n}\left(D_{n}^{\mathrm{BR}}(X ; \mathbb{Z})\right) \subset D_{n}^{\mathrm{BR} \mathrm{U}}(X ; \mathbb{Z})$.

Let $\boldsymbol{z}=\left(\boldsymbol{g} ; \boldsymbol{x}^{i-1}, 0, \boldsymbol{x}_{i+1}\right)$ be an element of $D_{n}^{\mathrm{BR}}(X ; \mathbb{Z})$. Put $a_{k}:=\sum_{j=k}^{n} m_{j}$. Then we have $a_{n}=m_{n}$ and $a_{k}-a_{k+1}=m_{k}$ for all $1 \leq k \leq n-1$. Moreover, $a_{i}=a_{i+1}$. Then, we have

$$
\gamma_{n}\left(\left(a_{1}, g_{1}\right), \ldots,\left(a_{n}, g_{n}\right)\right)=\boldsymbol{z} \in D_{n}^{\mathrm{BR}}(X ; \mathbb{Z}) .
$$

Let $\boldsymbol{z}=\left(\boldsymbol{g}^{i-1}, g_{i} h, \boldsymbol{g}_{i+1} ; \boldsymbol{x}\right)-(\boldsymbol{g} ; \boldsymbol{x})-\left(g_{i}^{-1} \boldsymbol{g}^{i-1} g_{i}, h, \boldsymbol{g}_{i+1} ; \boldsymbol{x}^{i-1} g_{i}, \boldsymbol{x}_{i} \varphi\left(g_{i}\right)\right) \in$ $D_{n}^{\mathrm{BR}}(X ; \mathbb{Z})$. Put $a_{k}:=\sum_{j=k}^{n} m_{j}$. Then we have $a_{n}=m_{n}$ and $a_{k}-a_{k+1}=m_{k}$ for all $1 \leq k \leq n-1$. Define $x_{k}:=\left(a_{k}, g_{k}\right)$ and $\boldsymbol{x}:=\left(x_{1}, \ldots, x_{n}\right)$. Then, we have

$$
\begin{aligned}
\gamma_{n}\left(\left(\boldsymbol{x}^{i-1},\left(a_{i}, g_{i} h\right), \boldsymbol{x}_{i+1}\right)\right. & -\left(\boldsymbol{x}^{i-1},\left(a_{i}, g_{i}\right), \boldsymbol{x}_{i+1}\right) \\
& \left.-\left(\boldsymbol{x}^{i-1} \underline{*}\left(a_{i}, g_{i}\right),\left(\left(a_{i}, h\right), \boldsymbol{x}_{i+1}\right) \bar{*}\left(a_{i}, g_{i}\right)\right)\right)=\boldsymbol{z} .
\end{aligned}
$$

Hence $\gamma_{n}^{-1}\left(D_{n}^{\mathrm{BR}}(X ; \mathbb{Z})\right) \subset D_{n}^{\mathrm{BR}}(X ; \mathbb{Z})$. This completes the proof.

\subsubsection{The induced homomorphism $\psi_{n, \lambda}$}

We fix a group homomorphism $\lambda: G \rightarrow A$. We also define a map $\tilde{\lambda}: C_{n}^{\mathrm{BR}_{\mathrm{U}}}(X ; \mathbb{Z}) \rightarrow$ $A$ as $\tilde{\lambda}\left(g_{1}, \ldots, g_{n} ; \boldsymbol{m}\right):=\lambda\left(g_{1}\right)$. In addition, we define a map $\psi_{n, \lambda}: C_{n}^{\mathrm{BR}}(X ; \mathbb{Z}) \rightarrow$ $C_{n}^{\mathrm{gp}}(M ; \mathbb{Z})_{G} \otimes A$ by $\psi_{n, \lambda}(\boldsymbol{g} ; \boldsymbol{m}):=\psi_{n}(\boldsymbol{g} ; \boldsymbol{m}) \otimes \tilde{\lambda}(\boldsymbol{g} ; \boldsymbol{m})$ for any integer $n \geq 2$.

Lemma 5.4. It holds that $\psi_{n, \lambda}\left(D_{n}^{\mathrm{BR}}(X ; \mathbb{Z})\right) \subset D_{n}^{\mathrm{gp}}(M ; \mathbb{Z})_{G} \otimes A$. Therefore, $\psi_{n, \lambda}$ induces the homomorphism

$$
\psi_{n, \lambda}: C_{n}^{\text {norBR }}(X ; \mathbb{Z}) \rightarrow C_{n}^{\text {norgp }}(M ; \mathbb{Z})_{G} \otimes A .
$$


Proof. It suffices to show that $\psi_{n, \lambda}\left(\boldsymbol{g} ; \boldsymbol{m}^{i-1}, 0, \boldsymbol{m}_{i+1}\right) \in D_{n}^{\mathrm{gp}}(M ; \mathbb{Z})_{G} \otimes A$ for $i$ with $1 \leq i \leq n-1$ and $\psi_{n, \lambda}\left(\boldsymbol{z}_{i}\right) \in D_{n}^{\mathrm{gp}}(M ; \mathbb{Z})_{G} \otimes A$ for $i$ with $1 \leq i \leq n$, where

$$
\boldsymbol{z}_{i}:=\left(\boldsymbol{g}^{i-1}, g_{i} h, \boldsymbol{g}_{i+1} ; \boldsymbol{m}\right)-(\boldsymbol{g} ; \boldsymbol{m})-\left(g_{i}^{-1} \boldsymbol{g}^{i-1} g_{i}, h, \boldsymbol{g}_{i+1} ; \boldsymbol{m}^{i-1} g_{i}, \boldsymbol{m}_{i} \varphi\left(g_{i}\right)\right) \text {. }
$$

We have

$\psi_{n}\left(\boldsymbol{g} ; \boldsymbol{m}_{i-1}, 0, \boldsymbol{m}_{i+1}\right)$

$=\sum_{\boldsymbol{k} \in \mathcal{K}^{n}}(-1)^{|\boldsymbol{k}|}\left(m_{1} \boldsymbol{g}_{\boldsymbol{k}_{1}}, \ldots, m_{i-1} \boldsymbol{g}_{\boldsymbol{k}_{i-1}}, 0, m_{i+1} \boldsymbol{g}_{\boldsymbol{k}_{i+1}}, \ldots, m_{n} \boldsymbol{g}_{\boldsymbol{k}_{n}}\right) \in D_{n}^{\mathrm{gp}}(M ; \mathbb{Z})_{G}$.

This inclusion $\psi_{n}\left(\boldsymbol{g} ; \boldsymbol{m}^{i-1}, 0, \boldsymbol{m}_{i+1}\right) \in D_{n}^{\mathrm{gp}}(M ; \mathbb{Z})_{G}$ implies $\psi_{n, \lambda}\left(\boldsymbol{g} ; \boldsymbol{m}^{i-1}, 0, \boldsymbol{m}_{i+1}\right) \in$ $D_{n}^{\mathrm{gp}}(M ; \mathbb{Z})_{G} \otimes A$.

Next, we show $\psi_{n, \lambda}\left(\boldsymbol{z}_{i}\right) \in D_{n}^{\mathrm{gp}}(M ; \mathbb{Z})_{G} \otimes A$.

For $i \neq 1$, we can prove that $\psi_{n}\left(\boldsymbol{z}_{i}\right)=0 \in D_{n}^{\mathrm{gp}}(M ; \mathbb{Z})_{G}$ as follows. Let $s$ be an integer with $1<s<i-1$. By the direct calculation, we have

$$
\begin{aligned}
& \psi_{n}\left(g_{i}^{-1} g_{1} g_{i}, \ldots, g_{i}^{-1} g_{i-1} g_{i}, h, g_{i+1}, \ldots, g_{n} ; m_{1} g_{i}, \ldots, m_{i-1} g_{i}, m_{i} \varphi\left(g_{i}\right), \ldots, m_{n} \varphi\left(g_{i}\right)\right) \\
& =\sum_{\boldsymbol{k} \in \mathcal{K}_{i}^{0}}(-1)^{|\boldsymbol{k}|}\left(m_{1} \varphi\left(g_{1}^{k_{1}}\right) g_{2}^{k_{2}} \cdots g_{i-1}^{k_{i-1}} g_{i} h^{0} g_{i+1}^{k_{i+1}} \cdots g_{n}^{k_{n}}, \ldots,\right. \\
& m_{s} \varphi\left(g_{1}^{k_{1}} \cdots g_{s}^{k_{s}}\right) g_{s+1}^{k_{s+1}} \cdots g_{i-1}^{k_{i-1}} g_{i} h^{0} g_{i+1}^{k_{i+1}} \cdots g_{n}^{k_{n}}, \ldots, \\
& m_{i-1} \varphi\left(g_{1}^{k_{1}} \cdots g_{i-1}^{k_{i-1}}\right) g_{i} h^{0} g_{i+1}^{k_{i+1}} \cdots g_{n}^{k_{n}} \\
& m_{i} \varphi\left(g_{1}^{k_{1}} \cdots g_{i-1}^{k_{i-1}} g_{i} h^{0}\right) g_{i+1}^{k_{i+1}} \cdots g_{n}^{k_{n}}, \ldots, \\
& \left.m_{n} \varphi\left(g_{1}^{k_{1}} \cdots g_{i-1}^{k_{i-1}} g_{i} h^{0} g_{i+1}^{k_{i+1}} \cdots g_{n}^{k_{n}}\right)\right) \\
& +\sum_{\boldsymbol{k} \in \mathcal{K}_{i}^{1}}(-1)^{|\boldsymbol{k}|}\left(m_{1} \varphi\left(g_{1}^{k_{1}}\right) g_{2}^{k_{2}} \cdots g_{i-1}^{k_{i-1}} g_{i} h^{1} g_{i+1}^{k_{i+1}} \cdots g_{n}^{k_{n}}, \ldots,\right. \\
& m_{s} \varphi\left(g_{1}^{k_{1}} \cdots g_{s}^{k_{s}}\right) g_{s+1}^{k_{s+1}} \cdots g_{i-1}^{k_{i-1}} g_{i} h^{1} g_{i+1}^{k_{i+1}} \cdots g_{n}^{k_{n}}, \ldots, \\
& m_{i-1} \varphi\left(g_{1}^{k_{1}} \cdots g_{i-1}^{k_{i-1}}\right) g_{i} h^{1} g_{i+1}^{k_{i+1}} \cdots g_{n}^{k_{n}} \\
& m_{i} \varphi\left(g_{1}^{k_{1}} \cdots g_{i-1}^{k_{i-1}} g_{i} h^{1}\right) g_{i+1}^{k_{i+1}} \cdots g_{n}^{k_{n}}, \ldots, \\
& \left.m_{n} \varphi\left(g_{1}^{k_{1}} \cdots g_{i-1}^{k_{i-1}} g_{i} h^{1} g_{i+1}^{k_{i+1}} \cdots g_{n}^{k_{n}}\right)\right) \text {. }
\end{aligned}
$$

We also have

$$
\begin{aligned}
& \psi_{n}\left(g_{1}, \ldots, g_{i-1}, g_{i}, g_{i+1}, \ldots, g_{n} ; m_{1}, \ldots, m_{n}\right) \\
& =\sum_{\boldsymbol{k} \in \mathcal{K}_{i}^{0}}(-1)^{|\boldsymbol{k}|}\left(m_{1} \varphi\left(g_{1}^{k_{1}}\right) g_{2}^{k_{2}} \cdots g_{i-1}^{k_{i-1}} g_{i}^{0} g_{i+1}^{k_{i+1}} \cdots g_{n}^{k_{n}}, \ldots,\right. \\
& m_{s} \varphi\left(g_{1}^{k_{1}} \cdots g_{s}^{k_{s}}\right) g_{s+1}^{k_{s+1}} \cdots g_{i-1}^{k_{i-1}} g_{i}^{0} g_{i+1}^{k_{i+1}} \cdots g_{n}^{k_{n}}, \ldots, \\
& m_{i-1} \varphi\left(g_{1}^{k_{1}} \cdots g_{i-1}^{k_{i-1}}\right) g_{i}^{0} g_{i+1}^{k_{i+1}} \cdots g_{n}^{k_{n}} \text {, } \\
& m_{i} \varphi\left(g_{1}^{k_{1}} \cdots g_{i-1}^{k_{i-1}} g_{i}^{0}\right) g_{i+1}^{k_{i+1}} \cdots g_{n}^{k_{n}}, \ldots, \\
& \left.m_{n} \varphi\left(g_{1}^{k_{1}} \cdots g_{i-1}^{k_{i-1}} g_{i}^{0} g_{i+1}^{k_{i+1}} \cdots g_{n}^{k_{n}}\right)\right) \\
& +\sum_{\boldsymbol{k} \in \mathcal{K}_{i}^{1}}(-1)^{|\boldsymbol{k}|}\left(m_{1} \varphi\left(g_{1}^{k_{1}}\right) g_{2}^{k_{2}} \cdots g_{i-1}^{k_{i-1}} g_{i}^{1} g_{i+1}^{k_{i+1}} \cdots g_{n}^{k_{n}}, \ldots,\right. \\
& m_{s} \varphi\left(g_{1}^{k_{1}} \cdots g_{s}^{k_{s}}\right) g_{s+1}^{k_{s+1}} \cdots g_{i-1}^{k_{i-1}} g_{i}^{1} g_{i+1}^{k_{i+1}} \cdots g_{n}^{k_{n}}, \ldots, \\
& m_{i-1} \varphi\left(g_{1}^{k_{1}} \cdots g_{i-1}^{k_{i-1}}\right) g_{i}^{1} g_{i+1}^{k_{i+1}} \cdots g_{n}^{k_{n}} \text {, } \\
& m_{i} \varphi\left(g_{1}^{k_{1}} \cdots g_{i-1}^{k_{i-1}} g_{i}^{1}\right) g_{i+1}^{k_{i+1}} \cdots g_{n}^{k_{n}}, \ldots, \\
& \left.m_{n} \varphi\left(g_{1}^{k_{1}} \cdots g_{i-1}^{k_{i-1}} g_{i}^{1} g_{i+1}^{k_{i+1}} \cdots g_{n}^{k_{n}}\right)\right) \text {. }
\end{aligned}
$$


Since (25) $=-(221)$, we have

$$
\begin{aligned}
& \psi_{n}\left(g_{1}, \ldots, g_{i-1}, g_{i} h, g_{i+1}, \ldots, g_{n} ; m_{1}, \ldots, m_{n}\right) \\
& =(23)+(24) \\
& =(22)+23)+(24)+25) .
\end{aligned}
$$

Then, we have $\psi_{n}\left(\boldsymbol{z}_{i}\right)=0 \in D_{n}^{\mathrm{gp}}(M ; \mathbb{Z})_{G}$.

Therefore, we have $\psi_{n, \lambda}\left(\boldsymbol{z}_{i}\right)=0 \in D_{n}^{\mathrm{gp}}(M ; \mathbb{Z})_{G} \otimes A$.

For the case that $i=1$, we have

$$
\begin{aligned}
& \psi_{n}\left(h, g_{2}, \ldots, g_{n} ; m_{1} \varphi\left(g_{1}\right), \ldots, m_{n} \varphi\left(g_{1}\right)\right) \\
& =\sum_{\boldsymbol{k} \in \mathcal{K}_{n}}(-1)^{|\boldsymbol{k}|}\left(m_{1} \varphi\left(g_{1}\right) \varphi\left(h^{k_{1}}\right) g_{2}^{k_{2}} \cdots g_{n}^{k_{n}}, \ldots, m_{n} \varphi\left(g_{1}\right) \varphi\left(h^{k_{1}} g_{2}^{k_{2}} \cdots g_{n}^{k_{n}}\right)\right) \\
& =\sum_{\boldsymbol{k} \in \mathcal{K}_{n}}(-1)^{|\boldsymbol{k}|}\left(m_{1} \varphi\left(h^{k_{1}}\right) g_{2}^{k_{2}} \cdots g_{n}^{k_{n}}, \ldots, m_{n} \varphi\left(h^{k_{1}} g_{2}^{k_{2}} \cdots g_{n}^{k_{n}}\right)\right) \\
& =\sum_{\boldsymbol{k} \in \mathcal{K}_{n}}(-1)^{|\boldsymbol{k}|}\left(m_{1} \varphi\left(\left(g_{1} h\right)^{k_{1}}\right) g_{2}^{k_{2}} \cdots g_{n}^{k_{n}}, \ldots, m_{n} \varphi\left(\left(g_{1} h\right)^{k_{1}} g_{2}^{k_{2}} \cdots g_{n}^{k_{n}}\right)\right) \\
& =\psi_{n}\left(g_{1} h, g_{2}, \ldots, g_{n} ; m_{1}, \ldots, m_{n}\right) \\
& =\psi_{n}\left(g_{1}, g_{2}, \ldots, g_{n} ; m_{1}, \ldots, m_{n}\right)
\end{aligned}
$$

since $k_{1}=0$. Then, we have

$$
\begin{aligned}
& \psi_{n, \lambda}\left(g_{1} h, g_{2}, \ldots, g_{n} ; m_{1}, \ldots, m_{n}\right) \\
& =\psi_{n}\left(g_{1} h, g_{2}, \ldots, g_{n} ; m_{1}, \ldots, m_{n}\right) \otimes \lambda\left(g_{1} h\right) \\
& =\psi_{n}\left(g_{1} h, g_{2}, \ldots, g_{n} ; m_{1}, \ldots, m_{n}\right) \otimes\left(\lambda\left(g_{1}\right)+\lambda(h)\right) \\
& =\psi_{n}\left(g_{1}, g_{2}, \ldots, g_{n} ; m_{1}, \ldots, m_{n}\right) \otimes \lambda\left(g_{1}\right) \\
& \quad+\psi_{n}\left(h, g_{2}, \ldots, g_{n} ; m_{1} \varphi\left(g_{1}\right), \ldots, m_{n} \varphi\left(g_{1}\right)\right) \otimes \lambda(h) \\
& =\psi_{n, \lambda}\left(g_{1}, g_{2}, \ldots, g_{n} ; m_{1}, \ldots, m_{n}\right)+\psi_{n, \lambda}\left(h, g_{2}, \ldots, g_{n} ; m_{1} \varphi\left(g_{1}\right), \ldots, m_{n} \varphi\left(g_{1}\right)\right) .
\end{aligned}
$$

Then, we have $\psi_{n, \lambda}\left(\boldsymbol{z}_{i}\right)=0 \in D_{n}^{\mathrm{gp}}(M ; \mathbb{Z})_{G} \otimes A$.

\subsection{Cocycles of $G$-Alexander MCB with the trivial $X$-set}

Let $X=\sqcup_{m \in M}(\{m\} \times G)$ be the $G$-Alexander multiple conjugation biquandle of $(M, \varphi)$. We consider the chain complex $C_{*}(X ; \mathbb{Z})$ defined in Subsection 2.4. where the $X$-set $Y$ is the trivial $X$-set. Thus the first element of $C_{n}(X ; \mathbb{Z})$ is omitted. For example, an element $\langle y\rangle\langle a\rangle\langle b\rangle-\langle y\rangle\langle a, a b\rangle+\langle y\rangle\langle b, a b\rangle \in C_{2}(X ; \mathbb{Z})$ is written by $\langle a\rangle\langle b\rangle-\langle a, a b\rangle+\langle b, a b\rangle$ for simplicity.

Define a homomorphism $\operatorname{proj}_{n}: P_{n}(X ; \mathbb{Z}) \rightarrow C_{n}^{\mathrm{BR}}(X ; \mathbb{Z})$ by

$$
\operatorname{proj}_{n}\left(\left\langle\boldsymbol{x}_{1}\right\rangle \cdots\left\langle\boldsymbol{x}_{k}\right\rangle\right)= \begin{cases}\left(x_{11}, x_{21}, \ldots, x_{k 1}\right) & (k=n) \\ 0 & \text { (otherwise) }\end{cases}
$$

for $n \geq 2$, where $\left\langle\boldsymbol{x}_{j}\right\rangle$ means $\left\langle x_{j 1}, x_{j 2}, \ldots, x_{j n_{j}}\right\rangle$ for each $j \in\{1, \ldots, k\}$. We define $\operatorname{proj}_{n}=0$ for $n \leq 1$.

Lemma 5.5. It holds that $\operatorname{proj}_{n}\left(D_{n}(X ; \mathbb{Z})\right) \subset D_{n}^{\mathrm{BR}}(X ; \mathbb{Z})$. 
Proof. Let $\left(m_{1}, g_{1}\right), \ldots,\left(m_{n}, g_{n}\right)$ be elements of $X$ such that $m_{i}=m_{i+1}$ for some $i$. Put $x_{i}:=\left(m_{i}, g_{j}\right)$. We show that

$$
\begin{aligned}
\operatorname{proj}_{n}( & \left\langle x_{1}\right\rangle \cdots\left\langle x_{i-1}\right\rangle\left\langle x_{i}\right\rangle\left\langle x_{i+1}\right\rangle\left\langle x_{i+2}\right\rangle \cdots\left\langle x_{n}\right\rangle- \\
& \left.\left\langle x_{1}\right\rangle \cdots\left\langle x_{i-1}\right\rangle\left\langle\left\langle x_{i}\right\rangle\left\langle x_{i+1}\right\rangle\right\rangle\left\langle x_{i+2}\right\rangle \cdots\left\langle x_{n}\right\rangle\right) \in D_{n}^{\mathrm{BR}}(X ; \mathbb{Z}) .
\end{aligned}
$$

We have

$$
\begin{aligned}
& \operatorname{proj}_{n}\left(\left\langle x_{1}\right\rangle \cdots\left\langle x_{i-1}\right\rangle\left\langle x_{i}\right\rangle\left\langle x_{i+1}\right\rangle\left\langle x_{i+2}\right\rangle \cdots\left\langle x_{n}\right\rangle\right. \\
& \left.\quad-\left\langle x_{1}\right\rangle \cdots\left\langle x_{i-1}\right\rangle\left\langle\left\langle x_{i}\right\rangle\left\langle x_{i+1}\right\rangle\right\rangle\left\langle x_{i+2}\right\rangle \cdots\left\langle x_{n}\right\rangle\right) \\
& =\operatorname{proj}_{n}\left(\left\langle x_{1}\right\rangle \cdots\left\langle x_{i-1}\right\rangle\left\langle x_{i}\right\rangle\left\langle x_{i+1}\right\rangle\left\langle x_{i+2}\right\rangle \cdots\left\langle x_{n}\right\rangle\right. \\
& \left.\quad-\left\langle x_{1}\right\rangle \cdots\left\langle x_{i-1}\right\rangle\left(\left\langle x_{i}, x_{i} x_{i+1}\right\rangle-\left\langle x_{i+1}, x_{i} x_{i+1}\right\rangle\right)\left\langle x_{i+2}\right\rangle \cdots\left\langle x_{n}\right\rangle\right) \\
& =\operatorname{proj}_{n}\left(\left\langle x_{1}\right\rangle \cdots\left\langle x_{i-1}\right\rangle\left\langle x_{i}\right\rangle\left\langle x_{i+1}\right\rangle\left\langle x_{i+2}\right\rangle \cdots\left\langle x_{n}\right\rangle\right) \\
& \quad-\operatorname{proj}_{n}\left(\left\langle x_{1}\right\rangle \cdots\left\langle x_{i-1}\right\rangle\left\langle x_{i}, x_{i} x_{i+1}\right\rangle\left\langle x_{i+2}\right\rangle \cdots\left\langle x_{n}\right\rangle\right) \\
& \quad+\operatorname{proj}_{n}\left(\left\langle x_{1}\right\rangle \cdots\left\langle x_{i-1}\right\rangle\left\langle x_{i+1}, x_{i} x_{i+1}\right\rangle\left\langle x_{i+2}\right\rangle \cdots\left\langle x_{n}\right\rangle\right) \\
& =\operatorname{proj}_{n}\left(\left\langle x_{1}\right\rangle \cdots\left\langle x_{i-1}\right\rangle\left\langle x_{i}\right\rangle\left\langle x_{i+1}\right\rangle\left\langle x_{i+2}\right\rangle \cdots\left\langle x_{n}\right\rangle\right) \\
& =\operatorname{proj}_{n}\left(\left\langle\left(m_{1}, g_{1}\right)\right\rangle \cdots\left\langle\left(m_{i}, g_{i}\right)\right\rangle\left\langle\left(m_{i}, g_{i+1}\right)\right\rangle \cdots\left\langle\left(m_{n}, g_{n}\right)\right\rangle\right) \\
& =\left(\left(m_{1}, g_{1}\right), \cdots,\left(m_{i}, g_{i}\right),\left(m_{i}, g_{i+1}\right), \cdots,\left(m_{n}, g_{n}\right)\right) \in D_{n}^{\mathrm{BR}}(X ; \mathbb{Z}) .
\end{aligned}
$$

We have $\operatorname{proj}_{n}\left(D_{n}(X ; \mathbb{Z})\right) \subset D_{n}^{\mathrm{BR}}(X ; \mathbb{Z})$.

Therefore, the homomorphism $\operatorname{proj}_{n}: P_{n}(X ; \mathbb{Z}) \rightarrow C_{n}^{\mathrm{BR}}(X ; \mathbb{Z})$ induces the homomorphism $\operatorname{proj}_{n}: C_{n}(X ; \mathbb{Z}) \rightarrow C_{n}^{\text {norBR }}(X ; \mathbb{Z})$.

Lemma 5.6. The map proj: $C_{*}(X ; \mathbb{Z}) \rightarrow C_{*}^{\text {norBR }}(X ; \mathbb{Z})$ is a chain map, that is, it holds that for any integer $n$

$$
\operatorname{proj}_{n-1} \circ \partial_{n}=\partial_{n}^{\text {norBR }} \circ \operatorname{proj}_{n} .
$$

Proof. It is sufficient to consider $\left\langle x_{1}\right\rangle \cdots\left\langle x_{n}\right\rangle$ in $C_{n}(X ; \mathbb{Z})$. For $\left\langle x_{1}\right\rangle \cdots\left\langle x_{n}\right\rangle$, we have

$$
\begin{aligned}
& \partial_{n}^{\text {norBR }} \circ \operatorname{proj}_{n}\left(\left\langle x_{1}\right\rangle \cdots\left\langle x_{n}\right\rangle\right) \\
& =\partial_{n}^{\text {norBR }}\left(x_{1}, \ldots, x_{n}\right) \\
& =\sum_{i=1}^{n}(-1)^{i-1}\left\{\left(\boldsymbol{x}^{i-1}, \boldsymbol{x}_{i+1}\right)-\left(\boldsymbol{x}^{i-1} \underline{*} x_{i}, \boldsymbol{x}_{i+1} \bar{*} x_{i}\right)\right\} \\
& =\sum_{i=1}^{n}(-1)^{i-1}\left\{\operatorname{proj}_{n-1}\left(\left\langle x_{1}\right\rangle \cdots\left\langle x_{i-1}\right\rangle\left\langle x_{i+1}\right\rangle \cdots\left\langle x_{n}\right\rangle\right)\right. \\
& \left.-\operatorname{proj}_{n-1}\left(\left\langle x_{1} \underset{*}{*} x_{i}\right\rangle \cdots\left\langle x_{i-1} \underline{*} x_{i}\right\rangle\left\langle x_{i+1} \mp x_{i}\right\rangle \cdots\left\langle x_{n} \bar{*} x_{i}\right\rangle\right)\right\} \\
& =\operatorname{proj}_{n-1} \circ \partial_{n}\left(\left\langle x_{1}\right\rangle \cdots\left\langle x_{n}\right\rangle\right) \text {. }
\end{aligned}
$$

We have $\operatorname{proj}_{n-1} \circ \partial_{n}=\partial_{n}^{\text {norBR }} \circ \operatorname{proj}_{n}$.

Fix a group homomorphism $\lambda: G \rightarrow A$. As a consequence of Lemma 5.6 and Subsection 5.1, we have the sequence

$$
C_{n}(X ; \mathbb{Z}) \stackrel{\text { proj }_{n}}{\longrightarrow} C_{n}^{\text {norBR }}(X ; \mathbb{Z}) \stackrel{\gamma_{n}}{\longrightarrow} C_{n}^{\text {norBR }_{\mathrm{U}}}(X ; \mathbb{Z}) \stackrel{\psi_{n, \lambda}}{\longrightarrow} C_{n}^{\text {norgp }}(M ; \mathbb{Z})_{G} \otimes A
$$

of chain groups. Therefore, we have the following theorem. 
Theorem 5.7. For any $n$-cocycle $f: C_{n}^{\operatorname{norgp}}(M ; \mathbb{Z})_{G} \rightarrow A$, the map

$$
\Phi_{f, \lambda}:=\left(f \otimes \operatorname{id}_{A}\right) \circ \psi_{n, \lambda} \circ \gamma_{n} \circ \operatorname{proj}_{n}: C_{n}(X ; \mathbb{Z}) \rightarrow A
$$

is an $n$-cocycle of the $G$-Alexander $M C B X=\sqcup_{m \in M}(\{x\} \times G)$.

The following theorem follows from the direct calculation.

Theorem 5.8. (1) Let $f: M^{2} \rightarrow A$ be a $G$-invariant A-multilinear map. The 2-cocycle $\Phi_{f, \lambda}=\left(f \otimes \mathrm{id}_{A}\right) \circ \psi_{2, \lambda} \circ \gamma_{2} \circ \operatorname{proj}_{2}: C_{2}(X ; \mathbb{Z}) \rightarrow A$ of the G-Alexander $M C B X=\bigsqcup_{m \in M}(\{m\} \times G)$ is formulated as

$$
\begin{aligned}
& \quad \Phi_{f, \lambda}\left(\left\langle\left(m_{1}, g_{1}\right)\right\rangle\left\langle\left(m_{2}, g_{2}\right)\right\rangle\right)=f\left(m_{1}-m_{2}, m_{2}\left(1-\varphi\left(g_{2}\right) g_{2}^{-1}\right)\right) \otimes \lambda\left(g_{1}\right) \\
& \text { for }\left\langle\left(m_{1}, g_{1}\right)\right\rangle\left\langle\left(m_{2}, g_{2}\right)\right\rangle \in X^{2} \subset C_{2}(X ; \mathbb{Z}) .
\end{aligned}
$$

(2) Let $f: M^{3} \rightarrow A$ be a G-invariant A-multilinear map. The 3-cocycle $\Phi_{f, \lambda}=\left(f \otimes \operatorname{id}_{A}\right) \circ \psi_{3, \lambda} \circ \gamma_{3} \circ \operatorname{proj}_{3}: C_{3}(X ; \mathbb{Z}) \rightarrow A$ of the $G$-Alexander $M C B X=\bigsqcup_{m \in M}(\{m\} \times G)$ is formulated as

$$
\begin{aligned}
& \quad \Phi_{f, \lambda}\left(\left\langle\left(m_{1}, g_{1}\right)\right\rangle\left\langle\left(m_{2}, g_{2}\right)\right\rangle\left\langle\left(m_{3}, g_{3}\right)\right\rangle\right) \\
& \quad=f\left(\left(m_{1}-m_{2}\right)\left(1-\varphi\left(g_{2}\right)^{-1} g_{2}\right), m_{2}-m_{3}, m_{3}\left(1-\varphi\left(g_{3}\right) g_{3}^{-1}\right)\right) \otimes \lambda\left(g_{1}\right) \\
& \text { for }\left\langle\left(m_{1}, g_{1}\right)\right\rangle\left\langle\left(m_{2}, g_{2}\right)\right\rangle\left\langle\left(m_{3}, g_{3}\right)\right\rangle \in X^{3} \subset C_{3}(X ; \mathbb{Z}) .
\end{aligned}
$$

\section{Cocycles of $G$-Alexander multiple conjugation biquandles with the $X$-set $X$}

Throughout this section, let $X=\bigsqcup_{m \in M}(\{m\} \times G)=M \times G$ the $G$-Alexander multiple conjugation biquandle of $(M, \varphi)$. We assume that the $X$-set $Y$ is $X$ itself. Our goal in this section is to give Theorem 6.8

\subsection{Degenerate subcomplexes $D_{*}^{\mathrm{BR}}(X ; \mathbb{Z})_{X}, D_{*}^{\mathrm{BR}_{\mathrm{U}}}(X ; \mathbb{Z})_{X}$ and the induced homomorphisms $\gamma_{n}, \psi_{n}$ and $\psi_{n, \lambda}$}

6.1.1 The degenerate subcomplex $D_{*}^{\mathrm{BR}}(X ; \mathbb{Z})_{X}$ of $C_{*}^{\mathrm{BR}}(X ; \mathbb{Z})_{X}$

Let $D_{n}^{\mathrm{BR}}(X ; \mathbb{Z})_{X}$ be the subgroup of $C_{n}^{\mathrm{BR}}(X ; \mathbb{Z})_{X}$ generated by the elements of the following sets

$$
\begin{aligned}
& \bigcup_{i=1}^{n-1}\left\{\left(\boldsymbol{x}^{i-1},(m, g),(m, h), \boldsymbol{x}_{i+2}\right) \mid \boldsymbol{x} \in X \times X^{n}, m \in M, g, h \in G\right\} \text { and } \\
& \bigcup_{i=1}^{n}\left\{\begin{array}{ll}
\left(\boldsymbol{x}^{i-1},(m, g h), \boldsymbol{x}_{i+1}\right)-\left(\boldsymbol{x}^{i-1},(m, g), \boldsymbol{x}_{i+1}\right) & \boldsymbol{x} \in X \times X^{n}, \\
-\left(\boldsymbol{x}^{i-1} \underline{*}(m, g),\left((m, h), \boldsymbol{x}_{i+1}\right) \bar{*}(m, g)\right) & m \in M, g, h \in G
\end{array}\right\}
\end{aligned}
$$

for $n \geq 2$, where we write

$$
\begin{aligned}
& \left(\boldsymbol{x}^{i-1} \text { * }(m, g),\left((m, h), \boldsymbol{x}_{i+1}\right) \bar{*}(m, g)\right) \\
& =\left(x_{0} \underline{*}(m, g), \ldots, x_{i-1} \underline{*}(m, g),(m, h) \text { * }(m, g), x_{i+1} \bar{*}(m, g), \ldots, x_{n} \approx(m, g)\right) .
\end{aligned}
$$

Define $D_{n}^{\mathrm{BR}}(X ; \mathbb{Z})_{X}:=0$ for $n \leq 1$. 
Lemma 6.1. $D_{*}^{\mathrm{BR}}(X ; \mathbb{Z})_{X}:=\left(D_{n}^{\mathrm{BR}}(X ; \mathbb{Z})_{X}, \partial_{n}^{\mathrm{BR}}\right)_{n \in \mathbb{Z}}$ is a subcomplex of $C_{*}^{\mathrm{BR}}(X ; \mathbb{Z})_{X}$.

The chain complex

$$
C_{*}^{\mathrm{norBR}}(X ; \mathbb{Z})_{X}:=C_{*}^{\mathrm{BR}}(X ; \mathbb{Z})_{X} / D_{*}^{\mathrm{BR}}(X ; \mathbb{Z})_{X}
$$

determines the homology group $H_{n}^{\text {norBR }}(X ; \mathbb{Z})_{X}$. In the ordinary way, for an abelian group $A$, we have the (co)homology theory with the coefficient group $A$ and the homology group $H_{n}^{\text {norBR }}(X ; A)_{X}$ and the cohomology group $H_{\text {norBR }}^{n}(X ; A)_{X}$ are defined.

\subsubsection{The degenerate subcomplex $D_{*}^{\mathrm{BR}}(X ; \mathbb{Z})_{X}$ of $C_{*}^{\mathrm{BR}_{\mathrm{U}}}(X ; \mathbb{Z})_{X}$}

Let $D_{n}^{\mathrm{BR}}(X ; \mathbb{Z})_{X}$ be the subgroup of $C_{n}^{\mathrm{BR}_{\mathrm{U}}}(X ; \mathbb{Z})_{X}$ generated by the elements of the following sets

$$
\begin{aligned}
& \bigcup_{i=1}^{n-1}\left\{\left(\boldsymbol{g} ; \boldsymbol{m}^{i-1}, 0, \boldsymbol{m}_{i+1}\right) \mid \boldsymbol{g} \in G \times G^{n}, \boldsymbol{m} \in M \times M^{n}\right\} \text { and } \\
& \bigcup_{i=1}^{n}\left\{\begin{array}{l|l}
\left(\boldsymbol{g}^{i-1}, g_{i} h, \boldsymbol{g}_{i+1} ; \boldsymbol{m}\right)-(\boldsymbol{g} ; \boldsymbol{m}) & h \in G, \boldsymbol{g} \in G \times G^{n}, \\
-\left(g_{i}^{-1} \boldsymbol{g}^{i-1} g_{i}, h, \boldsymbol{g}_{i+1} ; \boldsymbol{m}^{i-1} g_{i}, \boldsymbol{m}_{i} \varphi\left(g_{i}\right)\right) & \boldsymbol{m} \in M \times M^{n}
\end{array}\right\}
\end{aligned}
$$

for $n \geq 2$, where we write

$$
\begin{gathered}
\left(\boldsymbol{g} ; \boldsymbol{m}^{i-1}, 0, \boldsymbol{m}_{i+1}\right):=\left(g_{0}, g_{1}, \ldots, g_{n} ; m_{0}, m_{1}, \ldots, m_{i-1}, 0, m_{i+1}, \ldots, m_{n}\right), \\
\left(\boldsymbol{g}^{i-1}, g_{i} h, \boldsymbol{g}_{i+1} ; \boldsymbol{m}\right):=\left(g_{0}, g_{1}, \ldots, g_{i-1}, g_{i} h, g_{i+1}, \ldots, g_{n} ; m_{0}, m_{1}, \ldots, m_{n}\right) \text { and } \\
\left(g_{i}^{-1} \boldsymbol{g}^{i-1} g_{i}, h, \boldsymbol{g}_{i+1} ; \boldsymbol{m}^{i-1} g_{i}, \boldsymbol{m}_{i} \varphi\left(g_{i}\right)\right):=\left(\left(g_{i}^{-1} g_{0} g_{i}\right),\left(g_{i}^{-1} g_{1} g_{i}\right), \ldots,\left(g_{i}^{-1} g_{i-1} g_{i}\right),\right. \\
\left.\quad h, g_{i+1}, \ldots, g_{n} ; m_{0} g_{i}, m_{1} g_{i}, \ldots, m_{i-1} g_{i}, m_{i} \varphi\left(g_{i}\right), \ldots, m_{n} \varphi\left(g_{i}\right)\right) .
\end{gathered}
$$

Define $D_{n}^{\mathrm{BR}_{\mathrm{U}}}(X ; \mathbb{Z})_{X}:=0$ for $n \leq 1$.

Lemma 6.2. $D_{*}^{\mathrm{BR}}(X ; \mathbb{Z})_{X}:=\left(D_{n}^{\mathrm{BR}_{\mathrm{U}}}(X ; \mathbb{Z})_{X}, \partial_{n}^{\mathrm{BR}_{\mathrm{U}}}\right)_{n \in \mathbb{Z}}$ is a subcomplex of $C_{*}^{\mathrm{BR}_{\mathrm{U}}}(X ; \mathbb{Z})_{X}$.

The chain complex

$$
C_{*}^{\mathrm{norBR}_{\mathrm{U}}}(X ; \mathbb{Z})_{X}:=C_{*}^{\mathrm{BR}}(X ; \mathbb{Z})_{X} / D_{*}^{\mathrm{BR}}(X ; \mathbb{Z})_{X}
$$

determines the homology group $H_{n}^{\text {norBRu }}(X ; \mathbb{Z})_{X}$. In the ordinary way, for an abelian group $A$, we have the (co)homology theory with the coefficient group $A$ and the homology group $H_{n}^{\text {norBR }}(X ; A)_{X}$ and the cohomology group $H_{\text {norBR }}^{n}(X ; A)_{X}$ are defined.

\subsubsection{The induced homomorphism $\gamma_{n}$}

Next lemma shows that the isomorphism $\gamma_{n}: C_{n}^{\mathrm{BR}}(X ; \mathbb{Z})_{X} \rightarrow C_{n}^{\mathrm{BR} U}(X ; \mathbb{Z})_{X}$ defined in Subsection 4.1 induces the isomorphism $\gamma_{n}: C_{n}^{\text {norBR }}(X ; \mathbb{Z})_{X} \rightarrow$ $C_{n}^{\text {norBR }}(X ; \mathbb{Z})_{X}$, where we denote it by the same symbol $\gamma_{n}$ for simplicity.

Lemma 6.3. It holds that $\gamma_{n}\left(D_{n}^{\mathrm{BR}}(X ; \mathbb{Z})_{X}\right)=D_{n}^{\mathrm{BR}}(X ; \mathbb{Z})_{X}$. Therefore $\gamma_{n}$ induces the isomorphism

$$
\gamma_{n}: C_{n}^{\operatorname{norBR}}(X ; \mathbb{Z})_{X} \rightarrow C_{n}^{\text {norBR }_{U}}(X ; \mathbb{Z})_{X}
$$




\subsubsection{The induced homomorphisms $\psi_{n}$ and $\psi_{n, \lambda}$}

Next lemma shows that the map $\psi_{n}: C_{n}^{\mathrm{BR}}(X ; \mathbb{Z})_{X} \rightarrow C_{n+1}^{\mathrm{gp}}(M ; \mathbb{Z})_{G}$ defined in Subsection 4.1 induces the homomorphism $\psi_{n}: C_{n}^{\text {norBR }}(X ; \mathbb{Z})_{X} \rightarrow$ $C_{n+1}^{\text {norgp }}(M ; \mathbb{Z})_{G}$, where we denote it by the same symbol $\psi_{n}$ for simplicity.

Lemma 6.4. It holds that $\psi_{n}\left(D_{n}^{\mathrm{BR}}(X ; \mathbb{Z})_{X}\right) \subset D_{n+1}^{\mathrm{gp}}(M ; \mathbb{Z})_{G}$. Therefore $\psi_{n}$ induces the homomorphism

$$
\psi_{n}: C_{n}^{\text {norBR }_{\mathrm{U}}}(X ; \mathbb{Z})_{X} \rightarrow C_{n+1}^{\text {norgp }}(M ; \mathbb{Z})_{G}
$$

In addition, a homomorphism $\psi_{n, \lambda}: C_{n}^{\text {norBR }}(X ; \mathbb{Z})_{X} \rightarrow C_{n+1}^{\text {norgp }}(M ; \mathbb{Z})_{G} \otimes A$ is also defined as follows: We fix a group homomorphism $\lambda: G \rightarrow A$. We define a $\operatorname{map} \tilde{\lambda}: C_{n}^{\mathrm{BR}}(X ; \mathbb{Z})_{X} \rightarrow A$ as $\tilde{\lambda}\left(g_{0}, g_{1}, \ldots, g_{n} ; m_{0}, m_{1}, \ldots, m_{n}\right)=\lambda\left(g_{0}\right)$. Define a map $\psi_{n, \lambda}: C_{n}^{\mathrm{BR}_{\mathrm{U}}}(X ; \mathbb{Z})_{X} \rightarrow C_{n+1}^{\mathrm{gp}}(M ; \mathbb{Z})_{G} \otimes A$ by $\psi_{n, \lambda}=\psi_{n} \otimes \tilde{\lambda}$ for $n \geq 1$. Define $\psi_{n, \lambda}:=0$ for $n<1$.

Lemma 6.5. It holds that $\psi_{n, \lambda}\left(D_{n}^{\mathrm{BR}}(X ; \mathbb{Z})_{X}\right) \subset D_{n+1}^{\mathrm{gp}}(M ; \mathbb{Z})_{G} \otimes A$. Therefore $\psi_{n, \lambda}$ induces the homomorphism

$$
\psi_{n, \lambda}: C_{n}^{\mathrm{norBR}_{\mathrm{U}}}(X ; \mathbb{Z})_{X} \rightarrow C_{n+1}^{\mathrm{norgp}}(M ; \mathbb{Z})_{G} \otimes A
$$

\subsection{Cocycles of $G$-Alexander MCB with the $X$-set $X$}

Define a homomorphism $\operatorname{proj}_{n}: P_{n}(X ; \mathbb{Z})_{X} \rightarrow C_{n}^{\mathrm{BR}}(X ; \mathbb{Z})_{X}$ by

$$
\operatorname{proj}_{n}\left(\left\langle x_{0}\right\rangle\left\langle\boldsymbol{x}_{1}\right\rangle \cdots\left\langle\boldsymbol{x}_{k}\right\rangle\right)= \begin{cases}\left(x_{0}, x_{11}, \ldots, x_{k 1}\right) & (k=n) \\ 0 & \text { (otherwise) }\end{cases}
$$

for $n \geq 2$, where $\left\langle\boldsymbol{x}_{j}\right\rangle$ means $\left\langle x_{j 1}, x_{j 2}, \ldots, x_{j n_{j}}\right\rangle$ for each $j \in\{1, \ldots, k\}$. Define $\operatorname{proj}_{n}=0$ for $n \leq 1$.

Lemma 6.6. It holds that $\operatorname{proj}_{n}\left(D_{n}(X ; \mathbb{Z})_{X}\right) \subset D_{n}^{\mathrm{BR}}(X ; \mathbb{Z})_{X}$.

Therefore, the homomorphism $\operatorname{proj}_{n}: P_{n}(X ; \mathbb{Z})_{X} \rightarrow C_{n}^{\mathrm{BR}}(X ; \mathbb{Z})_{X}$ induces the homomorphism $\operatorname{proj}_{n}: C_{n}(X ; \mathbb{Z})_{X} \rightarrow C_{n}^{\text {norBR }}(X ; \mathbb{Z})_{X}$.

Lemma 6.7. The map proj is a chain map, that is, it holds that

$$
\operatorname{proj}_{n-1} \circ \partial_{n}=\partial_{n}^{\text {norBR }} \circ \operatorname{proj}_{n} .
$$

As a consequence of Lemma 6.7 and Subsection 6.1. we have two sequences

$$
\begin{aligned}
& C_{n}(X ; \mathbb{Z})_{X} \stackrel{\text { Proj }_{n}}{\longrightarrow} C_{n}^{\text {norBR }}(X ; \mathbb{Z})_{X} \stackrel{\gamma_{n}}{\longrightarrow} C_{n}^{\text {norBR }_{\mathrm{U}}}(X ; \mathbb{Z})_{X} \stackrel{\psi_{n}}{\longrightarrow} C_{n+1}^{\text {norgp }}(M ; \mathbb{Z})_{G} \otimes A, \\
& C_{n}(X ; \mathbb{Z})_{X} \stackrel{\text { proj }_{n}}{\longrightarrow} C_{n}^{\text {norBR }}(X ; \mathbb{Z})_{X} \stackrel{\gamma_{n}}{\longrightarrow} C_{n}^{\text {norBR }_{\mathrm{U}}}(X ; \mathbb{Z})_{X} \stackrel{\psi_{n, \lambda}}{\longrightarrow} C_{n+1}^{\text {norgp }}(M ; \mathbb{Z})_{G} \otimes A .
\end{aligned}
$$

Therefore, we have the following theorem.

Theorem 6.8. For any $(n+1)$-cocycle $f: C_{n+1}^{\text {norgp }}(M ; \mathbb{Z})_{G} \rightarrow A$, the maps

$$
\begin{aligned}
\Phi_{f} & :=f \circ \psi_{n} \circ \gamma_{n} \circ \operatorname{proj}_{n}: C_{n}(X ; \mathbb{Z})_{X} \rightarrow A \text { and } \\
\Phi_{f, \lambda} & :=\left(f \otimes \operatorname{id}_{A}\right) \circ \psi_{n, \lambda} \circ \gamma_{n} \circ \operatorname{proj}_{n}: C_{n}(X ; \mathbb{Z})_{X} \rightarrow A
\end{aligned}
$$

are $n$-cocycles of the $G$-Alexander $M C B X=\bigsqcup_{m \in M}(\{m\} \times G)$. 
Remark 6.9. The cocycle $\Phi_{f}$ does not use the information $g_{0}$ of $\left(m_{0}, g_{0}\right) \in$ $X=M \times G$. Therefore, we may replace the $X$-set $X=M \times G$ with $M$, that is, $\Phi_{f}$ can be also defined as the map from $C_{n}(X ; \mathbb{Z})_{X}$ to $A$.

Theorem 6.10. (1) Let $f: M^{3} \rightarrow A$ be a $G$-invariant A-multilinear map. The 2-cocycle $\Phi_{f}=f \circ \psi_{2} \circ \gamma_{2}: C_{2}(X ; \mathbb{Z})_{X} \rightarrow A$ of the $G$-Alexander $M C B$ $X=M \times G=\bigsqcup_{m \in M}(\{m\} \times G)$ is formulated as

$$
\begin{aligned}
& \Phi_{f}\left(\left\langle\left(m_{0}, g_{0}\right)\right\rangle\left\langle\left(m_{1}, g_{1}\right)\right\rangle\left\langle\left(m_{2}, g_{2}\right)\right\rangle\right) \\
& \quad=f\left(m_{0}^{\prime}\left(1-\varphi\left(g_{1}\right)^{-1} g_{1}\right), m_{1}^{\prime}, m_{2}\left(1-\varphi\left(g_{2}\right) g_{2}^{-1}\right)\right)
\end{aligned}
$$

for any $\left\langle\left(m_{0}, g_{0}\right)\right\rangle\left\langle\left(m_{1}, g_{1}\right)\right\rangle\left\langle\left(m_{2}, g_{2}\right)\right\rangle \in X \times X^{2}$, where $m_{i}^{\prime}:=m_{i}-m_{i+1}$.

(2) Let $f: M^{4} \rightarrow A$ be a G-invariant A-multilinear map. The 3-cocycle $\Phi_{f}=f \circ \psi_{3} \circ \gamma_{3}: C_{3}(X ; \mathbb{Z})_{X} \rightarrow A$ of the $G$-Alexander $M C B X=M \times G=$ $\bigsqcup_{m \in M}(\{m\} \times G)$ is formulated as

$$
\begin{aligned}
\Phi_{f} & \left(\left\langle\left(m_{0}, g_{0}\right)\right\rangle\left\langle\left(m_{1}, g_{1}\right)\right\rangle\left\langle\left(m_{2}, g_{2}\right)\right\rangle\left\langle\left(m_{3}, g_{3}\right)\right\rangle\right) \\
\quad & f\left(m_{0}^{\prime}\left(1-\varphi\left(g_{1}\right)^{-1} g_{1}\right), m_{1}^{\prime}, m_{2}^{\prime}, m_{3}\left(1-\varphi\left(g_{3}\right) g_{3}^{-1}\right)\right) \\
& \quad-f\left(m_{0}^{\prime}\left(1-\varphi\left(g_{1}\right)^{-1} g_{1}\right) g_{2}, m_{1}^{\prime} g_{2}, m_{2}^{\prime} \varphi\left(g_{2}\right), m_{3}\left(1-\varphi\left(g_{3}\right) g_{3}^{-1}\right) \varphi\left(g_{2}\right)\right)
\end{aligned}
$$

for any $\left\langle\left(m_{0}, g_{0}\right)\right\rangle\left\langle\left(m_{1}, g_{1}\right)\right\rangle\left\langle\left(m_{2}, g_{2}\right)\right\rangle\left\langle\left(m_{3}, g_{3}\right)\right\rangle \in X \times X^{3}$, where $m_{i}^{\prime}:=$ $m_{i}-m_{i+1}$.

Theorem 6.11. (1) Let $f: M^{3} \rightarrow A$ be a G-invariant A-multilinear map. The 2-cocycle $\Phi_{f, \lambda}=\left(f \otimes \mathrm{id}_{A}\right) \circ \psi_{2, \lambda} \circ \gamma_{2}: C_{2}(X ; \mathbb{Z})_{X} \rightarrow A$ of the $G$ Alexander $M C B X=\sqcup_{m \in M}(\{x\} \times G)$ is formulated as

$$
\begin{aligned}
& \Phi_{f, \lambda}\left(\left\langle\left(m_{0}, g_{0}\right)\right\rangle\left\langle\left(m_{1}, g_{1}\right)\right\rangle\left\langle\left(m_{2}, g_{2}\right)\right\rangle\right) \\
& =f\left(m_{0}^{\prime}\left(1-\varphi\left(g_{1}\right)^{-1} g_{1}\right), m_{1}^{\prime}, m_{2}\left(1-\varphi\left(g_{2}\right) g_{2}^{-1}\right)\right) \otimes \lambda\left(g_{0}\right)
\end{aligned}
$$

for any $\left\langle\left(m_{0}, g_{0}\right)\right\rangle\left\langle\left(m_{1}, g_{1}\right)\right\rangle\left\langle\left(m_{2}, g_{2}\right)\right\rangle \in X \times X^{2}$, where $m_{i}^{\prime}:=m_{i}-m_{i+1}$.

(2) Let $f: M^{4} \rightarrow A$ be a $G$-invariant A-multilinear map. The 3-cocycle $\Phi_{f, \lambda}=\left(f \otimes \operatorname{id}_{A}\right) \circ \psi_{3, \lambda} \circ \gamma_{3}: C_{3}(X ; \mathbb{Z})_{X} \rightarrow A$ of the $G$-Alexander $M C B$ $X=\sqcup_{m \in M}(\{x\} \times G)$ is formulated as

$$
\begin{aligned}
& \Phi_{f, \lambda}\left(\left\langle\left(m_{0}, g_{0}\right)\right\rangle\left\langle\left(m_{1}, g_{1}\right)\right\rangle\left\langle\left(m_{2}, g_{2}\right)\right\rangle\left\langle\left(m_{3}, g_{3}\right)\right\rangle\right) \\
& =f\left(m_{0}^{\prime}\left(1-\varphi\left(g_{1}\right)^{-1} g_{1}\right), m_{1}^{\prime}, m_{2}^{\prime}, m_{3}\left(1-\varphi\left(g_{3}\right) g_{3}^{-1}\right)\right) \otimes \lambda\left(g_{0}\right) \\
& \quad-f\left(m_{0}^{\prime}\left(1-\varphi\left(g_{1}\right)^{-1} g_{1}\right) g_{2}, m_{1}^{\prime} g_{2}, m_{2}^{\prime} \varphi\left(g_{2}\right), m_{3}\left(1-\varphi\left(g_{3}\right) g_{3}^{-1}\right) \varphi\left(g_{2}\right)\right) \otimes \lambda\left(g_{0}\right)
\end{aligned}
$$

for any $\left\langle\left(m_{0}, g_{0}\right)\right\rangle\left\langle\left(m_{1}, g_{1}\right)\right\rangle\left\langle\left(m_{2}, g_{2}\right)\right\rangle\left\langle\left(m_{3}, g_{3}\right)\right\rangle \in X \times X^{3}$, where $m_{i}^{\prime}:=$ $m_{i}-m_{i+1}$

\section{References}

[1] S. Carter, M. Elhamdadi and M. Saito, Homology theory for the settheoretic Yang-Baxter equation and knot invariants from generalizations of quandles, Fund. Math. 184 (2004), 31-54. 
[2] S. Carter, A. Ishii, M. Saito and K. Tanaka, Homology for quandles with partial group operations, Pacific J. Math. 287 (2017) 19-48.

[3] J. S. Carter, D. Jelsovsky, S. Kamada, L. Langford and M. Saito, Quandle cohomology and state-sum invariants of knotted curves and surfaces, Trans. Amer. Math. Soc. 355 (2003), 3947-3989.

[4] J. Ceniceros, M. Elhamdadi, M. Green and S. Nelson Augmented biracks and their homology, Internat. J. Math. 25 (2014), 1450087, 19 pp.

[5] R. Fenn, M. Jordan, and L. Kauffman, Biquandles and virtual links, Topology Appl. 145 (2004), 157-175.

[6] R. Fenn and C. Rourke, Racks and links in codimension two, J. Knot Theory Ramifications 1 (1992), 343-406.

[7] R. Fenn, C. Rourke and B. Sanderson, Trunks and classifying spaces, Appl. Categ. Structures 3 (1995), 321-356.

[8] R. Fenn, C. Rourke and B. Sanderson, The rack space, Trans. Amer. Math. Soc. 359 (2007), no. 2, 701-740.

[9] S. Eilenberg, S. MacLane, Cohomology theory in abstract groups. I, I, Ann. Math. 48 (1947), 51-78.

[10] A. Ishii, Moves and invariants for knotted handlebodies, Algebr. Geom. Topol. 8 (2008), 1403-1418.

[11] A. Ishii, A multiple conjugation quandle and handlebody-knots, Topology Appl. 196 (2015), 492-500.

[12] A. Ishii and M. Iwakiri, Y. Jang and K. Oshiro, A G-family of quandles and handlebody-knots, Illinois J. Math. 57 (2013), 817-838.

[13] A. Ishii, M. Iwakiri, S. KamadaJ. Kim, S. Matsuzaki and K. Oshiro, A multiple conjugation biquandles and handlebody-links, Hiroshima Math. J. 48 (2018), 89-117.

[14] A. Ishii, M. Iwakiri, S. KamadaJ. Kim, S. Matsuzaki and K. Oshiro, Biquandle (co)homology and handlebody-links, J. Knot Theory Ramifications 27 (2018), 1843001 (33pages).

[15] A. Ishii and S. Nelson, Partially multiplicative biquandles and handlebodyknots, Contemp. Math. 689 (2017), 159-176.

[16] D. Joyce, A classifying invariant of knots, the knot quandle, J. Pure Appl. Alg. 23 (1982), 37-65.

[17] L. H. Kauffman and D. E. Radford, Bi-oriented quantum algebras, and generalized Alexander polynomial for virtual links, Contemp. Math., 318 (2003), 113-140.

[18] V. Lebed, Knotted 3-Valent Graphs, Branched Braids, and MultiplicationConjugation Relations in a Group, Proceedings of Intelligence of LowDimensional Topology, RIMS Kokyuroku 1911 (2014), 86-100. 
[19] V. Lebed, Qualgebras and Knotted 3-Valent Graphs, Fund. Math. 230 (2015), no. 2, 167-204.

[20] S. V. Matveev, Distributive groupoids in knot theory, Mat. Sb. (N.S.) 119(161) (1982), 78-88.

[21] T. Nosaka, Quandle cocycles from invariant theory, Adv. Math. 245 (2013), 423-438. 